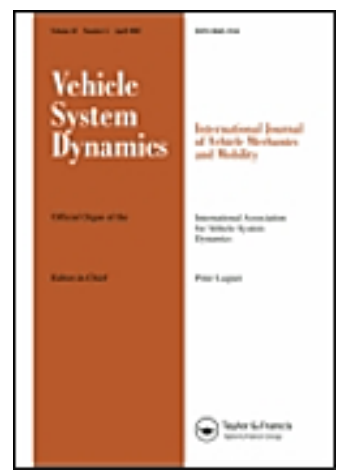

\title{
A cooperative control strategy for yaw rate and sideslip angle control combining Torque Vectoring with Rear Wheel Steering
}

\begin{tabular}{|c|c|}
\hline Journal: & Vehicle System Dynamics \\
\hline Manuscript ID & NVSD-2020-0104.R3 \\
\hline Manuscript Type: & Original Paper \\
\hline $\begin{array}{r}\text { Date Submitted by the } \\
\text { Author: }\end{array}$ & 22-Oct-2020 \\
\hline Complete List of Authors: & $\begin{array}{l}\text { Vignati, Michele; Politecnico di Milano, Mechanical Engineering } \\
\text { Sabbioni, Edoardo; Politecnico di Milano }\end{array}$ \\
\hline Keywords: & $\begin{array}{l}\text { Electric vehicles, Electronic stability control < Chassis control systems, } \\
\text { Nonlinear control < Control, } 4 \text { WS < Steering systems, Driver assistance } \\
\text { systems }\end{array}$ \\
\hline \multicolumn{2}{|c|}{$\begin{array}{l}\text { Note: The following files were submitted by the author for peer review, but cannot be converted to PDF. } \\
\text { You must view these files (e.g. movies) online. }\end{array}$} \\
\hline $\begin{array}{l}\text { 2019_NVSD_tv4ws_Final.tex } \\
\text { Sec_Control.tex } \\
\text { Sec_Symbols.tex }\end{array}$ & \\
\hline
\end{tabular}

\section{SCHOLARONE Manuscripts}




\title{
ARTICLE TEMPLATE
}

\section{A cooperative control strategy for yaw rate and sideslip angle control combining Torque Vectoring with Rear Wheel Steering}

\author{
M. Vignati and E. Sabbioni \\ Department of Mechanical Engineering, Politecnico di Milano, via La Masa 1, 20156, Milano \\ (MI), Italy
}

\section{ARTICLE HISTORY}

Compiled December 23, 2020

\begin{abstract}
Automobiles are becoming more and more complex as multiple control systems are integrated into the vehicle platform. This paper investigates the coordination of active rear steering (RWS) and torque vectoring (TV) - which is enabled by independent electric motors at the rear axle - in controlling vehicle lateral dynamics. Specifically, the proposed controller aims at enhancing vehicle handling performance and stability while cornering. The coordination of the two control systems is achieved by weighting their contribution based on their impact on vehicle dynamics according to the working condition. The impact of each control system is assessed by means of phase portraits. These plots are in fact a very powerful tool for analyzing vehicle nonlinear dynamics as they readily display vehicle stability properties and map equilibrium point locations and movement to changing parameters and control inputs. Based on phase portrait analysis, a performance index is thus proposed, which weights more the control action (TV or RWS) capable of leading the vehicle at the nearest equilibrium point with the fastest rate. The controller performance is assessed through numerical simulations carried out using a nonlinear 14 dofs vehicle model. Results are compared with ones of the two controllers alone (RWS and TV) in different cornering maneuvers and considering different adherence conditions.
\end{abstract}

\section{KEYWORDS}

torque vectoring, rear wheel steering, yaw rate control, sideslip control, electric motors, MIMO systems

\section{Introduction}

There is an increasing demand for enhancement of stability and handling performance in the automotive field. The presence of multiple actuators (such as active steering, active suspension, differential braking, etc.) increases the possibility of achieving this goal. Moreover, the shift from ICE (Internal Combustion Engine) to electric vehicles opens to additional control opportunities such as torque vectoring, which is enabled by independent electric motors $([1-18])$.

Lateral dynamics controls systems typically consist of a yaw rate reference follower or sideslip angle limiter; a feedback controller generates as outputs the required yaw moment or steer angle. Early papers on active rear steering have focused on reducing the vehicle sideslip angle, whereas more recent ones concentrate their attention on the 
yaw motion control [19] [16]. Canale et al. reported that it is necessary to control the sideslip angle along with the yaw motion to maintain the stability of a vehicle. A considerable amount of research has been conducted on the control of vehicle dynamics using yaw rate control, sideslip control, or a combination of the two [20]. In order to generate higher tire slip angles (i.e. higher lateral tire forces) some controllers cause higher vehicle sideslip angles, thus, yaw rate control and sideslip control should be considered together when designing a electronic stability controller [21] [22] [2]. In the lateral dynamics control two main aspects have to be considered: the vehicle stability and the maneuverability. Tchamna et al. [22] reported that the yaw rate control objective primarily concerns to the improvement of steering feel, while sideslip control is more related to vehicle stability and it is important near the limit of vehicle handling. The studies have moved along the implementation of multi-actuator systems, keeping in mind pros and cons of different control actuators [23-26]. In [27], [13], [14], [28] and [3] some PI based controls are proposed. Marino et al. [27], use a decentralized PI control to follow a first order non-linear model reference driven by the driver input. This control structure allows them to set arbitrary steady state values for lateral speed and yaw rate at any longitudinal speed.

The increasing complexity of vehicles - due to multiple actuators/controllers - calls for new tools helping system engineers to assess the impact of the additional control systems on vehicle safety and performance, as well as their integration with the existing ones. Phase portraits are proven to be a very effective tool for evaluating the impact of control actions on enhancing vehicle handling performance and stability [3]. These plots in fact provide a graphical illustration of vehicle nonlinear dynamics, which is particularly useful in systems, which are described by two dominant states, such as vehicles running a turn on a planar surface. As a matter of fact, while ground vehicles have many degrees of freedom, the fundamental planar instabilities critical to control problems such as yaw rate control or stabilizing a drifting vehicle arise from the yaw and sideslip dynamics $([18,29-31])$. In these cases, plotting the phase portraits allows to map equilibrium points locations and types and the range of possible trajectories in response to constrained or nonlinear control inputs. Phase portrait analysis is particularly informative when it is crucial accounting for nonlinear dynamics and it is difficult to display state trajectories from the equations of motion alone.

The present paper, in particular, proposes the use of phase portraits to integrate and coordinate the control actions of two different active systems: torque vectoring (TV), enabled by two independent electric motors placed at the rear axle, and rear wheel steering (RWS). Specifically, phase portraits are used study the impact of the two controllers on vehicle turning dynamics. Based on this analysis, a performance index is defined to assign a weight to each control action, according to the working condition. The largest weight is assigned to the controller showing the the highest capability of leading the vehicle at a stable equilibrium point. Other authors explored the stability of vehicle planar motion using phase plane portraits: Pacejka ([29]) states that "...For motions with constant steer angle (possibly after a step change), the system is autonomous and the phase-plane representation may be used to find the solution..." and continues with "...The isocline method turns out to be straightforward and simple to employ. The pattern of the trajectories is strongly influenced by the socalled singular points. In these points the motion finds an equilibrium. In the singular points the motion is stationary and consequently, the differentials of the state variables vanish...". In [32] Bobier showed the used of phase portrait for studying the vehicle dynamics for the design of active control systems. In [31] the phase plane is used to study the coupling between vehicle and driver. In [18] the phase plane is instead used 
to study the effect of torque vectoring in controlling vehicle drift equilibria.

This way seems to be the most promising since other methods suffer of different problems.

PIDs, for example, are the most used controllers. Goggia et al. [14] propose a control based on PID control structures with complex multiparametric gain scheduling. Xiong et al. [33] propose a gain scheduling approach to deal with the non-linearity of vehicle dynamics mainly derived from tyre/road adhesion characteristics. PIDs were born for dealing with linear systems and in fact the vehicle model adopted in these controllers is usually linearised about an equilibrium point. This makes it difficult to predict the behavior of the controller far from the equilibrium point; in particular when it moves due to system non-linearity.

Other authors used fuzzy logic to coordinate the two actuators. They defined membership functions that classify the system output as 'small' or 'large' and generate inputs accordingly. Fuzzy coordination is also very natural in the case where input sub-system controllers are themselves fuzzy systems; in this case the inputs may remain fuzzy variables, in the form of aggregated fuzzy sets; the coordinator can then performs additional aggregation, and defuzzification is only applied at the final step before actuation [25]. Fuzzy logic control is a knowledge-based control approach which can mimic human experience in controlling complex systems. Li ([24]) and Zaho ([34]) present fuzzy controllers applied to vehicle lateral dynamics. The problems of this type of controller is the correct definition of the fuzzy rules together with their tuning and the tuning of the important parameters of the system. This duty is, in general, demanded to the designer experience and his knowledge of the problem.

Another possibility, is to design integrated controllers via a full-vehicle reference model. Model Predictive Control (MPC) is most spreading method. It has some important advantages but also some criticisms. MPC exploits a model of the system dynamics to predict the future system evolution and to select the best control action accordingly to a specified performance criterion. As opposed to standard optimal control, in MPC the input trajectory is recomputed every time new information on the system becomes available. At every control cycle, MPC computes the solution of a finite horizon optimal control problem based on the system dynamics and operating constraints. Thus, a particular advantage of MPC is the capability of coordinating several constrained actuators to achieve multiple goals encoded in the performance criterion like done in $[3,23,35]$. Several other authors have proposed vehicles integrated control based on MPC ([15,36-39]). All of them have to balance computation cost and complexity of the model. In particular in more than one case the capability of MPC to deal with non-linearities are not completely exploited due to the difficulty to run it in real-time. The problem related to the definition of the prevision length and the time discretization is fundamental in fast system dynamics as in the vehicle case. Moreover, the state estimation of not directly measurable parameter occurs in the implementation of MPCs.

To overcome the limitations given by strong non linear dynamics, this work studies the coupling effect of TV and RWS by analyzing the vehicle dynamics in the phase plane adopting a non linear vehicle model. The realized phase plots are used to synthesize the performances of each actuator, i.e. TV and RWS, by highlighting the capability of each single actuator in modifying the vehicle dynamics. The results obtained are then used to design an active control system for controlling vehicle lateral dynamics by coordinating the two actuators as suggested by the results of the vehicle dynamics evaluation with phase plane.

The main benefits of the selected approach over the ones previously described are: 
(1) it accounts for vehicle non-linear dynamics. The phase-plane analysis is in fact carried out considering a 4-points vehicle model including load transfers, nonlinear contact forces (Pacejka's Magic Formulae are adopted), combined slip effects and actuator characteristics (motor torque vs. speed characteristic curve, saturation, etc.). Based on this analysis, a performance index is proposed and mapped to weight the contribution of the two actuators (TV and RWS) according to their effectiveness;

(2) the computational cost is relatively small since mapping of the performance index is carried out off-line in a pre-processing phase;

(3) weighting of the contributions provided by the two controllers is based on the capability of the actuators in modifying vehicle states.

Points 1) and 2) allow to increase the complexity of the vehicle model without affecting the online performance (computational cost) of the controller, which is a strong limit for online controllers, such as MPC and/or LQR. Point 3) may help in addressing one of the open issues of fuzzy controllers, which is the definition and the tuning of the rules driving the control action. As previously mentioned, these rules are in fact generally selected based on designer experience and tuned using genetic algorithms/neural networks [40]. Similarly, to sliding mode control, the proposed controller takes advantage of the phase plane to define the control action. However, with respect to sliding mode control it allows to account for actuator characteristics in the design phase, which is often a critical point for the application of sliding mode techniques to real cases [41-43].

The paper is then organized as follows: section 2 shows the characteristics of the vehicle that has been used to study the actuator coordination effects and as a target for the controller.

Section 3 reports the phase plane analysis that allows to deeply investigate the effectiveness of the two actuators, TV and RWS, thus providing important information that are used to schedule the controller gains.

Section 4 shows the details about the combined controller which is a hierarchical control that combines TV and RWS based on the effectiveness evaluated in phase plane.

Finally, section 5 reports the simulation results of some significant maneuvers. The results compare the passive vehicle with the combined control and two additional controlled vehicles, one with TV the other with RWS, to evaluate the effective increase in performances adduced by the coordination of the two actuators.

\section{Vehicle characteristics}

The vehicle considered, whose data are reported in table 1, is a formula SAE championship electric vehicle. It is a rear wheels drive vehicle (RWD) that presents two independent $\mathrm{AC}$ brushless electric motors (EM) for left and right rear wheels with a chain transmission; the EM data are reported in table 1. Thanks to its structure, the vehicle well lends itself to the implementation of the Torque Vectoring (TV). The electric motors can be described by its characteristic torque-speed curve and by a first order time lag transfer function. The car is also fitted with a rear steering system (RWS) that is actively controlled by an electric motor (EM) again modeled accounting for torque-speed characteristics and time constant of a first order time lag transfer function. 
Table 1. Vehicle data

\begin{tabular}{llrl}
\hline description & symbol & value & units \\
\hline total vehicle mass & $m$ & 346 & $\mathrm{~kg}$ \\
yaw moment of inertia & $J_{z}$ & 116 & $\mathrm{~kg} \mathrm{~m}{ }^{2}$ \\
front axis to c.o.g. distance & $l_{\mathrm{f}}$ & 756 & $\mathrm{~mm}$ \\
rear axis to c.o.g. distance & $l_{\mathrm{r}}$ & 920 & $\mathrm{~mm}$ \\
front semi-track width & $c_{\mathrm{f}}$ & 625 & $\mathrm{~mm}$ \\
rear semi-track width & $c_{\mathrm{r}}$ & 600 & $\mathrm{~mm}$ \\
tire rolling radius & $R_{w}$ & 250 & $\mathrm{~mm}$ \\
Motor nominal voltage & $V_{m, \text { mom }}$ & 72 & $\mathrm{~V}$ \\
Motor peak power & $P_{m, \max }$ & 15 & $\mathrm{~kW}$ \\
Motor peak torque & $T_{m, \max }$ & 70 & $\mathrm{Nm}$ \\
Motor maximum speed & $\omega_{m, \max }$ & 6000 & $\mathrm{rpm}$ \\
Motor transmission ratio & $\tau_{m}$ & $1: 4$ & - \\
Motor time constant & $\Upsilon_{e}$ & 10 & $\mathrm{~ms}$ \\
Rear steer maximum angle & $\delta_{r, \max }$ & 3 & $\mathrm{deg}$ \\
Rear steer nominal voltage & $V_{\delta, \text { nom }}$ & 24 & $\mathrm{~V}$ \\
Rear steer motor peak power & $P_{\delta, \max }$ & 209 & $\mathrm{~W}$ \\
Rear steer motor peak torque & $T_{\delta, \max }$ & 2.0 & $\mathrm{Nm}$ \\
Rear steer motor max speed & $\omega_{\delta, \max }$ & 4000 & $\mathrm{rpm}$ \\
Rear steer transmission ratio & $\tau_{\delta}$ & $1: 20$ & - \\
Rear steer time constant & $\Upsilon_{\delta}$ & 100 & $\mathrm{~ms}$ \\
\hline
\end{tabular}

The two different actuation systems (TV and RWS) are characterized by different bandwidths. In particular, the rear steering system is a rack-pinion system with a high transmission ratio (one order of magnitude higher with respect to the final gear ratio of the traction motors) needed to maintain the vehicle lightness.

\section{Phase plot analysis}

To assess the impact of TV and RWS on vehicle nonlinear dynamics, a phase portrait analysis is carried out. Aim of the analysis is the definition of a performance index able to weight the action of the two controllers according to the working condition. In particular, phase portraits are used to identify which controller has more capability to lead the vehicle at a stable equilibrium point and consequently assign it the highest weight. The section is organized as follows: the vehicle model used to obtain the phase portraits is presented first; then results of the phase portrait analysis are discussed and finally the performance index is introduced.

\subsection{Vehicle model for Phase portrait generation}

A nonlinear double track vehicle model is used to perform the phase portrait analysis. It accounts for tire combined slip and lateral load transfer to correctly evaluate the effect of torque vectoring and rear wheel steering during cornering.

Making reference to Figure 1, the double track motion is described by the following 


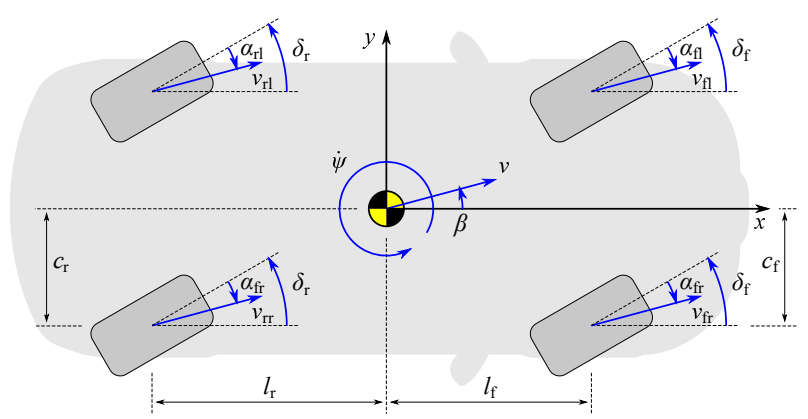

Figure 1. Vehicle model.

differential equations:

$$
\left\{\begin{array}{l}
m a_{x}=F_{x, f}+F_{x, r}-F_{r e s} \\
m a_{y}=F_{y, f}+F_{y, r} \\
J \ddot{\psi}=F_{y, f} l_{f}-F_{y, r} l_{r}+\Delta F_{x, f} c_{f}-\Delta F_{y, r} c_{r}+M_{z}
\end{array}\right.
$$

where $m$ is the vehicle mass, $J$ the yaw moment of inertia, $F_{\text {res }}$ the sum of aerodynamic resistance force and rolling resistance, $a_{x}$ and $a_{y}$ are respectively the longitudinal and lateral accelerations which are related to longitudinal speed and sideslip angle as follows

$$
\left\{\begin{array}{l}
v_{x}=v \cos \beta \\
v_{y}=v \sin \beta
\end{array}\right.
$$

and

$$
\left\{\begin{array}{l}
a_{x}=\dot{v}_{x}-\dot{\psi} v_{y}=\dot{v} \cos \beta-v \dot{\beta} \sin \beta-\dot{\psi} v \sin \beta \\
a_{y}=\dot{\psi} v_{x}+\dot{v}_{y}=\dot{\psi} v \cos \beta+\dot{v} \sin \beta+v \dot{\beta} \cos \beta
\end{array}\right.
$$

while

$$
\begin{aligned}
F_{x, f} & =\left(F_{x, f r}+F_{x, f l}\right) \cos \delta_{f}-\left(F_{y, f r}+F_{y, f l}\right) \sin \delta_{f} \\
F_{x, r} & =\left(F_{x, r r}+F_{x, r l}\right) \cos \delta_{r}-\left(F_{y, r r}+F_{y, r l}\right) \sin \delta_{r} \\
F_{y, f} & =\left(F_{x, f r}+F_{x, f l}\right) \sin \delta_{f}+\left(F_{y, f r}+F_{y, f l}\right) \cos \delta_{f} \\
F_{y, r} & =\left(F_{x, r r}+F_{x, r l}\right) \sin \delta_{r}+\left(F_{y, r r}+F_{y, r l}\right) \cos \delta_{r} \\
\Delta F_{x, f} & =\left(F_{x, f r}-F_{x, f l}\right) \cos \delta_{f}-\left(F_{y, f r}-F_{y, f l}\right) \sin \delta_{f} \\
\Delta F_{y, r} & =\left(F_{y, r r}-F_{y, r l}\right) \sin \delta_{r} \\
M_{z} & =\left(F_{x, r r}-F_{x, r l}\right) \cos \delta_{r}
\end{aligned}
$$

where $F_{x}$ and $F_{y}$ are the longitudinal and lateral forces. Subscripts $\bullet_{f}$ and $\bullet_{r}$ stand for front and rear respectively, while the four corners of the car are indicated as front right $\bullet_{f r}$, front left $\bullet_{f l}$, rear right $\bullet_{r r}$ and rear left $\bullet_{r l}$.

The tire-road contact forces are modeled according to Pacejka MF-tyre model in- 
cluding the effect of vertical load transfers

$$
F_{y_{0_{i}}}=D_{i} \sin \left(C_{i} \arctan \left(B_{i} \alpha_{i}-E_{i}\left(B_{i} \alpha_{i}-\arctan \left(B_{i} \alpha_{i}\right)\right)\right)\right)
$$

In which $\alpha_{i}$ is the tire slip angle defined as

$$
\begin{aligned}
& \alpha_{f r}=\delta_{f}-\arctan \frac{v_{y}+\dot{\psi} l_{f}}{v_{x}+\dot{\psi} c_{f}} \\
& \alpha_{f l}=\delta_{f}-\arctan \frac{v_{y}+\dot{\psi} l_{f}}{v_{x}-\dot{\psi} c_{f}} \\
& \alpha_{r r}=\delta_{r}-\arctan \frac{v_{y}-\dot{\psi} l_{r}}{v_{x}+\dot{\psi} c_{r}} \\
& \alpha_{r l}=\delta_{r}-\arctan \frac{v_{y}-\dot{\psi} l_{r}}{v_{x}-\dot{\psi} c_{r}}
\end{aligned}
$$

while

$$
D_{i}=\mu_{y i} F_{z i}\left(p_{1}+p_{2} d f_{z}\right)
$$

which highlights the dependency of lateral forces on the available friction coefficient in lateral direction $\mu_{y i}$ and the vertical force $F_{z i}$ as a non linear expression: $p_{1}$ and $p_{2}$ are constants while $d f_{z}$ is the normalized change in vertical force as defined in [29]. Vertical forces are dependent on steady state component of the load transfer which is a function of longitudinal and lateral accelerations:

$$
\begin{aligned}
& F_{z, f r}=\frac{m g l_{r}}{2}-\frac{a_{x} h_{G}}{l}+\frac{a_{y} h_{G} K_{\text {roll }}}{c_{f}} \\
& F_{z, f l}=\frac{m g l_{r}}{2}-\frac{a_{x} h_{G}}{l}-\frac{a_{y} h_{G} K_{\text {roll }}}{c_{f}} \\
& F_{z, r r}=\frac{m g l_{f}}{2}+\frac{a_{x} h_{G}}{l}+\frac{a_{y} h_{G}\left(1-K_{\text {roll }}\right)}{c_{r}} \\
& F_{z, r l}=\frac{m g l_{f}}{2}+\frac{a_{x} h_{G}}{l}-\frac{a_{y} h_{G}\left(1-K_{\text {roll }}\right)}{c_{r}}
\end{aligned}
$$

To account for the presence of longitudinal forces, some considerations have to be done. If no braking maneuvers are considered, the longitudinal forces at the front axle are null being the car RWD.

$\mu_{y i}$ is then the available friction coefficient that accounts for the residual friction coefficient due to longitudinal forces

$$
\mu_{y_{i}}=\sqrt{\mu^{2}-\left(\frac{F_{x_{i}}}{F_{z_{i}}}\right)^{2}}
$$

The rear longitudinal forces, which depend on both driver demand $F_{x, d}$ needed to 
maintain the vehicle speed and TV control input $\Delta F_{x, M z}$ :

$$
\begin{gathered}
F_{x r r}=\frac{F_{x, d}}{2}+\Delta F_{x, M z} \\
F_{x r l}=\frac{F_{x, d}}{2}-\Delta F_{x, M z}
\end{gathered}
$$

The longitudinal forces requested to generate the yaw moment are computed as

$$
\Delta F_{x, M z}=\frac{M_{z}}{c_{r}}
$$

$F_{x r}$ and $F_{x l}$ are saturated considering the friction limitation and electric motor maximum torque

$$
\begin{aligned}
& \left|F_{x r r}\right| \leq \min \left(\mu F_{z r r}, \frac{T_{m r}(v)}{R}\right) \\
& \left|F_{x r l}\right| \leq \min \left(\mu F_{z r l}, \frac{T_{m l}(v)}{R}\right)
\end{aligned}
$$

where $T_{m r}$ and $T_{m l}$ are the motor torques at the right and left rear wheel respectively which are function of vehicle speed, $R$ is the wheel rolling radius.

The full set of equations is solved (with a numerical solver in Matlab environment) and imposing:

- constant speed ( $v=$ constant, $\dot{v}=0$ which implies $a_{x} \approx 0$ );

- constant front steering angle $\left(\delta_{f}=\right.$ constant);

- constant rear wheel steering $\left(\delta_{r}=\right.$ constant) considering steering limits;

- constant uneven motor torques for TV yaw moment ( $M_{z}=$ constant) considering motor limits and friction limits;

- given initial conditions $\left(\beta_{0}, \dot{\psi}_{0}\right)$;

The outputs of the solver are the values of $\ddot{\psi}$ and $\dot{\beta}$ which are used to draw the phase portrait and to evaluate the controller performance indexes as described in the following.

When building the phase portrait for the passive vehicle both inputs $\delta_{r}$ and $M_{z}$ are considered null. To consider the effect of RWS several values of $\delta_{r}$ are considered while $M_{z}=0$. Viceversa, to consider the effect of TV, $M_{z}$ assumes different values while $\delta_{r}=0$.

\subsection{Phase portrait}

To handle the presence of multiple actuators some performance indexes are defined to weight each actuator contribution. The performance indexes are defined for each actuator by evaluating the capability of actuator to modify the vehicle dynamics, i.e. by evaluating the maximum yaw acceleration $\ddot{\psi}$ and the maximum sideslip angle rate $\dot{\beta}$ that can be provided by TV and RWS for each point in the phase plane $\left(\dot{\psi}_{0}, \beta_{0}\right)$. A phase portrait is then generated for each index.

A phase portrait graphically illustrates the dynamics of a system by plotting the state derivatives and resulting trajectories as a function of the state for fixed inputs. Phase portrait analysis has proved to be a very effective tool for analyzing the dynamics 


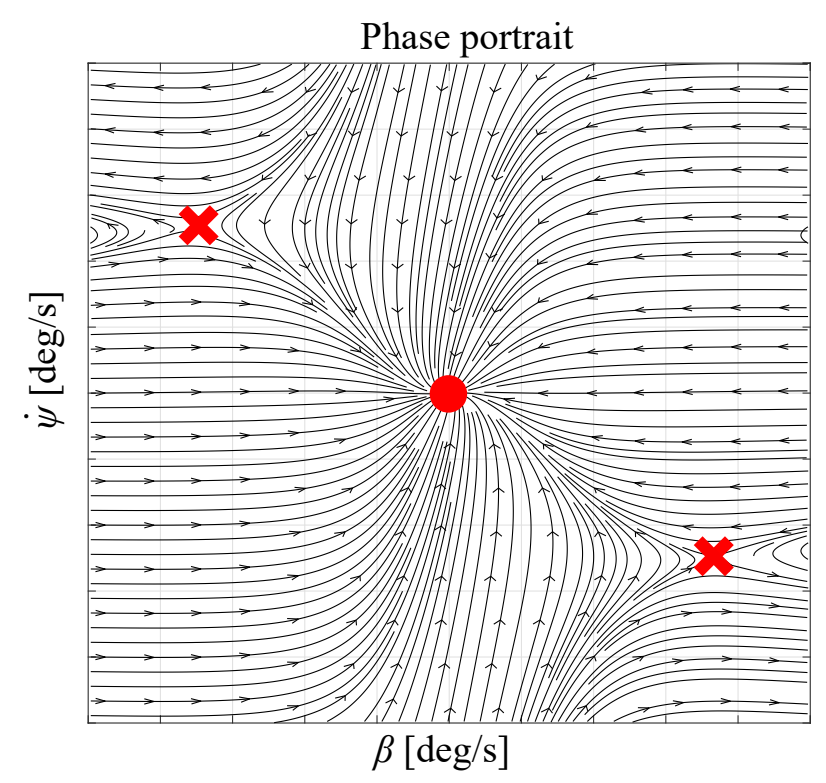

Figure 2. Phase portrait $\beta-\dot{\psi}$ of the vehicle at $60 \mathrm{~km} / \mathrm{h}$ and null steer angle.

of a nonlinear system as it immediately reveals the location and type of equilibria in the system and the region of attraction, or stable regions, about those equilibria.

$[18,29,31]$ showed that the fundamental planar instabilities critical to control problems such as ESP or stabilizing a drifting vehicle arise from the yaw and sideslip dynamics.

The equations of the vehicle model used to obtain the phase portrait are the ones presented in the previous section. As an example, figure 2 shows the phase portrait of the passive vehicle in straight driving $\left(\delta_{f}=0\right)$ at a speed $v=60 \mathrm{~km} / \mathrm{h}$ where the stable fixed point (dot) and saddle node (cross) are highlighted.

Notice that, although enlightening because of their visual nature, results of the phase portrait must be carefully analyzed to ensure that they are meaningful and consistent with the real dynamics of the vehicle. In fact, the phase portrait gives an instantaneous snapshot of the trajectories at each state combination for a predetermined set of parameters (e.g. vehicle speed or front steering angle) and a fixed control input (rear wheel steering or yawing moment). Therefore, the trajectory of the vehicle states can only be viewed as it would develop in time on single phase portrait only if steering and speed remain constant (or they are held constant of closed-loop control is applied to keep them constant). Otherwise, the motion in response to more general changes of speed, steering can only be found by redrawing the phase portrait at every speed and steer angle at each point in time. A set of maps is then necessary to consider a sufficient range of steering and speed values.

\subsection{Performance indexes definition}

As mentioned earlier, the main idea is to use phase plane portrait to weight the control actions provided by TV and RWS. Clearly the highest weight must be assigned to the controller showing the highest capability to lead the vehicle at a stable equilibrium point for the considered working condition. On this purpose, some performance indexes are introduced. These indexes represent the maximum increase (with sign) of yaw 

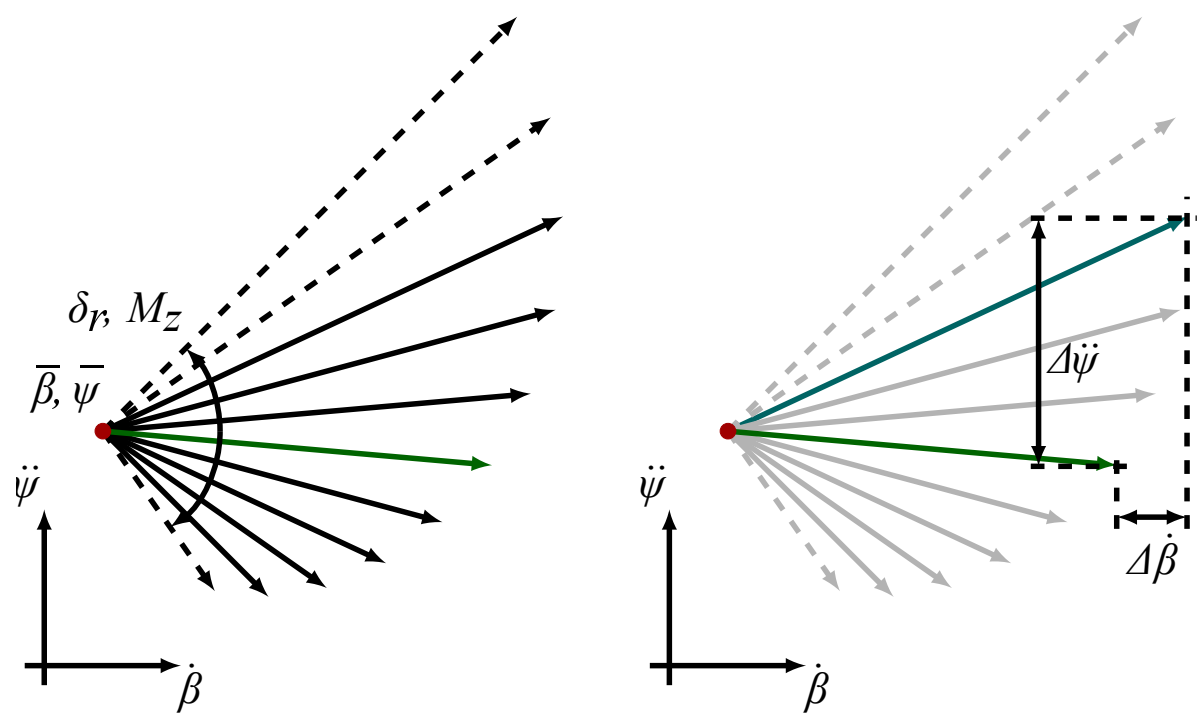

Figure 3. Performance indexes definition scheme.

acceleration and sideslip angle rate corresponding to a control input pair ( $\delta_{r}$ and $\left.M_{z}\right)$. Therefore, the highest is the performance indexes, the fastest is the rate with which the vehicle reaches the equilibrium point.

As better detailed in the following, the controller that uses the performance indexes is the sum of four PIDs each one using one actuator (TV or RWS) and each one tracking one reference (yaw rate or sideslip angle). This means that each PID performance is defined by yaw rate derivative or side slip angle derivative only.

More in details, the procedure for calculating the performance indexes is the following.

Following the map generation procedure, fixed the vehicle speed $v$, driver steering angle $\delta_{f}$, rear steering angle $\delta_{r}$ and yawing moment $M_{z}$ (i.e. the control inputs), the sideslip angle and yaw acceleration ( $\dot{\beta}$ and $\ddot{\psi}$ respectively) can be evaluated at each $\beta-\dot{\psi}$ combination. To study how much the two controllers are affecting the vehicle dynamics an iterative calculation varying the two control parameters $\left(\delta_{r}\right.$ and $\left.M_{z}\right)$ is performed.

The performance indexes are defined as the maximum improvement in positive and negative yaw acceleration $(\ddot{\psi})$ and sideslip angle derivative $(\dot{\beta})$ respectively. In other words, the best controller is the one heading the vehicle states to the stable equilibrium point at the fastest rate. For greater clarity, referring to figure 3, this concept can be represented. In the left scheme of figure 3 the vector in green represents the sideslip angle and yaw rate derivatives $(\dot{\beta}, \ddot{\psi})$ of the passive vehicle in a generic point $(\bar{\beta}, \bar{\psi})$ in the phase plane. The black vectors represent the sideslip angle and yaw rate derivatives $(\dot{\beta}, \ddot{\psi})$ associated with the different level of control action $\left(\delta_{r}\right.$ and $\left.M_{z}\right)$ with the solid ones which represent the only in feasible range.

The feasible region is defined by the actuators characteristics (maximum torque/angle) and system physical limits (i.e. the tyre-road friction coefficient). All the control inputs (TV and RWS) which cannot be provided by the actuators or producing a force exceeding the friction limit (see eq. 12) are not accounted for (the 
corresponding solution in terms of yaw acceleration and sideslip angle rate are not computed).

By selecting a generic solid one it is so possible to define the variation in sideslip angle derivative $(\Delta \dot{\beta})$ and in yaw-rate one $(\Delta \ddot{\psi})$ between the controlled vehicle, with a certain level of control action, and the passive one. The performance indexes associated to a $\bar{\beta}-\overline{\dot{\psi}}$ are defined as the maximum and minimum variation with sign of sideslip angle and yaw-rate derivatives in the feasibility region. As the aim is the comparison between the two kinds of actuation systems, the performance indexes are then normalized with respect to the maximum modulus of variation among all the performed evaluations.

The procedure to compute the performance index is as follows:

- define system inputs: front steering angle and forward speed values $u_{0}=\left(\delta_{0}, v_{0}\right)$;

- select one point in the phase plane $x_{0}=\left(\beta_{0}, \dot{\psi}_{0}\right)$;

- compute yaw rate derivative $(\ddot{\psi})$ for passive vehicle according to equations 1-12. Store the value as $\ddot{\psi}_{\text {pas }}\left(x_{0}, u_{0}\right)$;

- compute the yaw rate derivative $(\ddot{\psi})$ for all values of yaw moment by TV within motor/friction limits, store $\max$ and min value $\ddot{\psi}_{T V, \max }\left(x_{0}, u_{0}\right)$ and $\ddot{\psi}_{T V, \min }\left(x_{0}, u_{0}\right)$;

- compute the difference whit respect to passive

$$
\begin{aligned}
\Delta \ddot{\psi}_{T V+}\left(x_{0}, u_{0}\right) & =\ddot{\psi}_{T V, \max }\left(x_{0}, u_{0}\right)-\ddot{\psi}_{\text {pas }}\left(x_{0}, u_{0}\right) \\
\Delta \ddot{\psi}_{T V-}\left(x_{0}, u_{0}\right) & =\ddot{\psi}_{T V, \min }\left(x_{0}, u_{0}\right)-\ddot{\psi}_{\text {pas }}\left(x_{0}, u_{0}\right)
\end{aligned}
$$

- compute the yaw rate derivative for all values of rear steer within actuator limits, store max and min value $\ddot{\psi}_{R W S, \max }\left(x_{0}, u_{0}\right)$ and $\ddot{\psi}_{R W S, \min }\left(x_{0}, u_{0}\right)$;

- compute the difference with respect to passive

$$
\begin{aligned}
& \Delta \ddot{\psi}_{R W S+}\left(x_{0}, u_{0}\right)=\ddot{\psi}_{R W S, \max }\left(x_{0}, u_{0}\right)-\ddot{\psi}_{\text {pas }}\left(x_{0}, u_{0}\right) \\
& \Delta \ddot{\psi}_{R W S-}\left(x_{0}, u_{0}\right)=\ddot{\psi}_{R W S, \min }\left(x_{0}, u_{0}\right)-\ddot{\psi}_{\text {pas }}\left(x_{0}, u_{0}\right)
\end{aligned}
$$

- compute $\Delta \ddot{\psi}_{T V-}, \Delta \ddot{\psi}_{T V+}, \Delta \ddot{\psi}_{R W S_{-}}, \Delta \ddot{\psi}_{R W S+}$ values for all points in the phase plane;

- find $\max$ and $\min \Delta$ values:

$$
\begin{aligned}
\Delta \ddot{\psi}_{\max }\left(u_{0}\right) & =\max \left(\Delta \ddot{\psi}_{T V+}\left(\boldsymbol{x}, u_{0}\right), \Delta \ddot{\psi}_{R W S+}\left(\boldsymbol{x}, u_{0}\right)\right) \\
\Delta \ddot{\psi}_{\min }\left(u_{0}\right) & =\min \left(\Delta \ddot{\psi}_{T V-}\left(\boldsymbol{x}, u_{0}\right), \Delta \ddot{\psi}_{R W S-}\left(\boldsymbol{x}, u_{0}\right)\right)
\end{aligned}
$$


- normalize $\Delta$ values by the max and min obtaining the performance index

$$
\begin{aligned}
\chi_{11+}\left(\boldsymbol{x}, u_{0}\right) & =\frac{\Delta \ddot{\psi}_{T V+}\left(\boldsymbol{x}, u_{0}\right)}{\Delta \ddot{\psi}_{\max }\left(u_{0}\right)} \\
\chi_{11-}\left(\boldsymbol{x}, u_{0}\right) & =\frac{\Delta \ddot{\psi}_{T V-}\left(\boldsymbol{x}, u_{0}\right)}{\Delta \ddot{\psi}_{\min }\left(u_{0}\right)} \\
\chi_{21+}\left(\boldsymbol{x}, u_{0}\right) & =\frac{\Delta \ddot{\psi}_{R W S+}\left(\boldsymbol{x}, u_{0}\right)}{\Delta \ddot{\psi}_{\max }\left(u_{0}\right)} \\
\chi_{21-}\left(\boldsymbol{x}, u_{0}\right) & =\frac{\Delta \ddot{\psi}_{R W S-}\left(\boldsymbol{x}, u_{0}\right)}{\Delta \ddot{\psi}_{\min }\left(u_{0}\right)}
\end{aligned}
$$

- change inputs and compute new performance index;

- store all the values

- repeat the procedure for $\dot{\beta}$

\subsubsection{Performance indexes maps}

In figures 4 an examples of the obtained maps is proposed for positive increase of $\Delta \ddot{\psi}\left(\chi_{11+}\right.$ and $\left.\chi_{21+}\right)$ and $\Delta \dot{\beta}\left(\chi_{12+}\right.$ and $\left.\chi_{22+}\right)$. All the diagrams are superimposed to the passive vehicle phase portrait (white arrows) and the red circle identifies the equilibrium condition at the given speed and front steering angle. The red dashed line is the locus of the equilibria points varying the front steering angle. The colored contour shows the capability of the controller (TV or RWS) in modifying the yaw rate dynamics $(\Delta \ddot{\psi})$ or sideslip angle dynamics $(\Delta \dot{\beta})$ according to performance index that ranges from 0 to 1 . Yellow is associated with a performance index equal to 1 (i.e. the control action has the maximum effect in heading the vehicle at a stable equilibrium point), blue is associated with a performance index equal to 0 (i.e. the control action is not effective). Figure 4 refers to variations $\ddot{\psi}>0$ and $\dot{\beta}>0$. The maps for $\ddot{\psi}<0$ and $\dot{\beta}<0$ are in fact symmetric with respect to the $\mathrm{x}-\mathrm{y}$ axes.

The performance indexes $\chi$ are then defined as:

$\chi_{11}$ performance index of TV in modifying the yaw acceleration $(\ddot{\psi})$;

$\chi_{12}$ performance index of TV in modifying the sideslip angle derivative $(\dot{\beta})$;

$\chi_{21}$ performance index of RWS in modifying the yaw acceleration $(\ddot{\psi})$;

$\chi_{22}$ performance index of RWS in modifying the sideslip angle derivative $(\dot{\beta})$.

Once the controller is set, the performance indexes can be defined according to the 
tracking error and $\chi_{11+}, \chi_{11-}, \chi_{21+}, \chi_{21-}, \chi_{12+}, \chi_{12-}, \chi_{22+}, \chi_{22-}$ as follows

$$
\begin{aligned}
& \chi_{11}= \begin{cases}\chi_{11+} & \dot{\psi} \leq \dot{\psi}_{\text {ref }} \\
\chi_{11-} & \dot{\psi}>\dot{\psi}_{\text {ref }}\end{cases} \\
& \chi_{21}= \begin{cases}\chi_{21+} & \dot{\psi} \leq \dot{\psi}_{\text {ref }} \\
\chi_{21-} & \dot{\psi}>\dot{\psi}_{\text {ref }}\end{cases} \\
& \chi_{12}= \begin{cases}\chi_{12+} & \beta \leq \beta_{\text {ref }} \\
\chi_{12-} & \beta>\beta_{\text {ref }}\end{cases} \\
& \chi_{22}= \begin{cases}\chi_{22+} & \beta \leq \beta_{\text {ref }} \\
\chi_{22-} & \beta>\beta_{\text {ref }}\end{cases}
\end{aligned}
$$

The plots in left column represent the performance index associated to the torque vectoring $\left(\chi_{11}\right.$ and $\left.\chi_{12}\right)$, while the right ones refer to the rear wheel steering $\left(\chi_{21}\right.$ and $\chi_{22}$ ). The following sections draw the main conclusions in view of integrating the control actions.
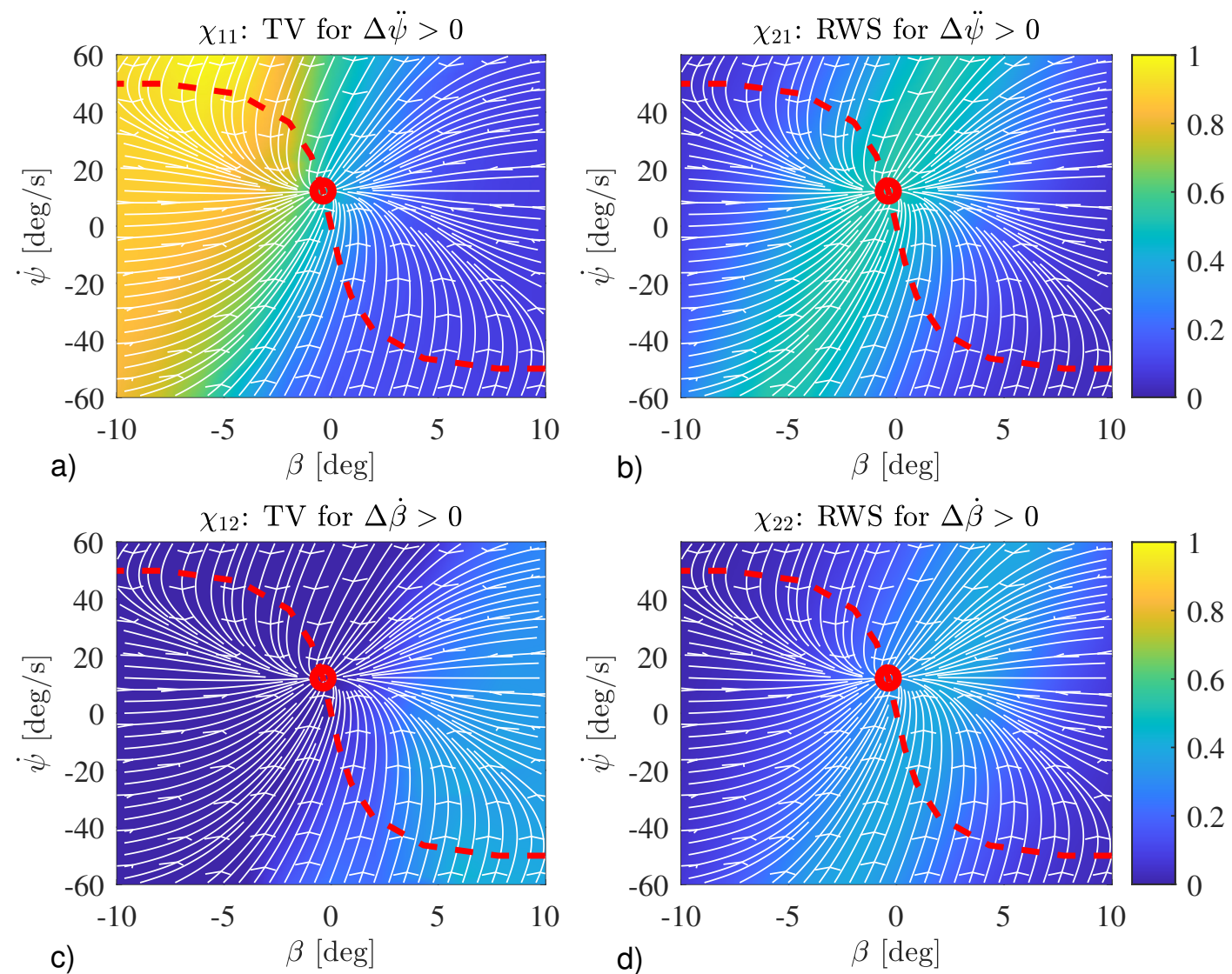

Figure 4. Performance indexes of TV and RWS when $v=50 \mathrm{~km} / \mathrm{h}$ and $\delta_{f}=1.5^{\circ}$. 


\subsubsection{Performance indexes for yaw rate control}

Considering the TV control action (Figure 4.a) it is possible to note an higher performance index on the left side of the maps in which the sideslip angle is negative. This denotes an higher capability in improving the vehicle yaw-rate when it has opposite sign of sideslip angle. Viceversa (yaw rate and sideslip angle of the same sign), the passive vehicle returns to the equilibrium position very fast, even without any control action. Although not shown in this paper, the performances of the control demonstrate its maximum capability to perturb the vehicle state for intermediate speeds. This is related to the high stability of the vehicle at low speeds; on the contrary at high speeds the performances are limited by the reduction of available motors torques and consequentially possible yawing moment.

Generally speaking, RWS has a lower impact on vehicle dynamics with respect to TV (Figure 4.b). This is mainly due to the limitation in the maximum steering angle which is fixed to 3 degrees. Although not reported here, the level of performances is not influenced by vehicle speed.

\subsubsection{Perfomance indexes for sideslip angle control}

In this case (Figure 4.c), the ability of TV in controlling sideslip angle denotes higher performance in the positive sideslip angle and negative yaw-rate zone. Contrarily to the yaw rate performance indexes, the RWS presents an higher impact with respect to TV and the same working zone. For what concerns the effect of different front steering angles on the indexes, it does not to affect the trend of the maps but the position of the equilibrium points around which the vehicle tends to work and to reduce their distance to the unstable points.

\subsection{Performance indexes map synthesis}

To correctly use the indexes previously defined the index maps have to be synthesized to account for a reasonable range of front steering angles and speed values.

To do this, several maps are generated for different front steering angles and different speeds values. For each map only a portion in the neighborhood of the equilibrium point is selected.

For each speed value is then possible to generate a performance index map that considers a number of front steering angle values. Figure 5 represents the performance indexes with respect to the yaw-rate derivative $\left(\chi_{11}\right.$ in top left and $\chi_{21}$ in top right diagrams) and sideslip angle derivative $\left(\chi_{12}\right.$ in bottom left and in $\chi_{22}$ bottom right diagrams) at a given speed $(50 \mathrm{~km} / \mathrm{h})$ and for discrete increase of the front steering angle $\left(\delta_{f}=0,1.5,3, \ldots 9^{\circ}\right)$. Each sub-rectangle is in fact built around the equilibrium point given by one value of front steering angle. For each performance index, 3 maps are generated according to speed values of 25,50 and $75 \mathrm{~km} / \mathrm{h}$.

For clarity only the results for one speed $(50 \mathrm{~km} / \mathrm{h})$ are reported. Moving from lowspeed maps to high-speed ones it is notable an higher performance of the RWS system in the range of limited sideslip angles. Especially for low speed, being the equilibrium points locus along the RWS maximum efficiency direction, the rear steering system presents higher effectiveness.

Focusing on Figures 5 (upper diagrams) it is visible a strong capability of the TV to move the yaw rate from 0 to the reference value (high capability to speed up the vehicle response to a steer variation), but a limited capability to decrease from higher 
yaw-rate to the reference ones is also present. Anyway, at high yaw-rate and sideslip angle values, it denotes higher performance indexes with respect to RWS.
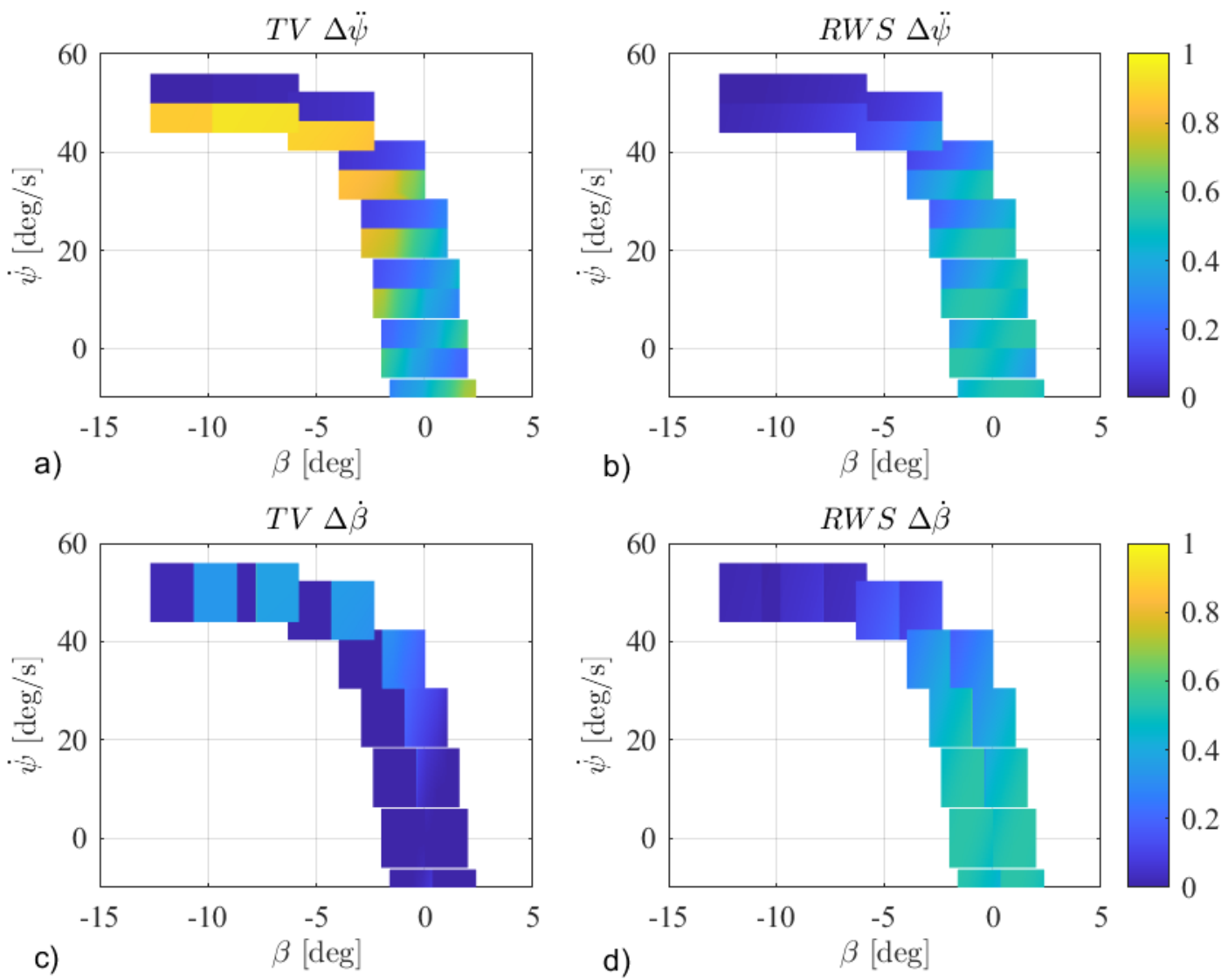

Figure 5. Combined maps of performance indexes of TV, RWS at $50 \mathrm{~km} / \mathrm{h}$.

The same kind of analysis is also performed on the capability of the actuator to perturb the sideslip angle. Figure 5 (lower diagrams) represents the performance indexes with respect to the sideslip angle derivative. In this case, the performance index maps are combined with respect to $\beta$ so the right side of the rectangles is associated to a maximization of the negative rate of the sideslip angle; the opposite happens on the left side. From Figure 5 the same limitations highlighted in the previous analysis are notable. Especially, even if the RWS demonstrates higher effectiveness with respect to the TV in a wider zone, the really limited capacity to manage the sideslip angle when it becomes too big opens to the possibility to coordinate the two controllers to handle these extreme zones. In general, the TV has demonstrated to be effective in a wider range, but for limited sideslip angle the more efficacious actuation system is the RWS.

The performance index maps allow to identify the working area of the two actuators. In particular, RWS is found to have poorer performances in controlling yaw rate with respect to TV as reported in Figure 5.a and 5.b. Viceversa, RWS capability in controlling sideslip angle is higher than TV in particular for in tire linear region (yaw rate far from friction limit) as reported in Figure 5.c and 5.d. TV capability in tracking sideslip angle increases instead when approaching the friction limit; this is probably due to combined slip condition. 


\begin{abstract}
torous
allocator
Figure 6. Control system architecture.
\end{abstract}

In parallel to the performances in the control of vehicle dynamics, the actuator bandwidth needs to be taken into consideration. In particular the torque vectoring control action is provided by the electric motor varying the longitudinal forces applied to the tires. On the other hand, RWS system manages the rear wheel steering angle changing the tire lateral forces and it is actuated by an electrical motor with a reduction ratio one order of magnitude higher with respect to the propulsion EMs. Moreover, tires dynamics response has to be considered. It depends on tire relaxation lengths that in general, is smaller in case of longitudinal forces. This makes the tire response faster in the longitudinal direction than in the lateral one. Due to the sum of these two effects, the TV is capable to generate faster control actions than the RWS system and this needs to be strongly considered in the design of the vehicle dynamics control logic.

\title{
4. Control strategy
}

The control strategy is a hierarchical control. In a first level the values of the references are defined and in the second one the control action evaluation is performed. Aiming at defining coordination between TV and RWS, a control action is referred to the rear yawing moment $M_{z}$ and the rear steering angle $\delta_{r}$. At the lower level, the implementation of an anti-slip control (ASC) and the control action allocation are performed.

The scheme of the multi-input multi-output (MIMO) controller is reported in Figure 6 . The yaw rate and sideslip angle references are evaluated according to the driver demands. Then, based on the phase portrait analysis and proposed performance indexes, the following controller is implemented, which combines:

- a PI regulator which defines $M_{z, \dot{\psi}}$ based on yaw rate reference;

- a PI regulator which defines $M_{z, \beta}$ based on side slip angle reference;

- a PI regulator which defines $\delta_{r, \dot{\psi}}$ based on yaw rate reference;

- a PI regulator which defines $\delta_{r, \beta}$ based on side slip reference;

Controller gains are scheduled based on the previously defined performance indexes 
$\chi$ so to weight more the controller which is more effective. Specifically, the rear yaw moment and the rear steering angle control action are multiplied by their performance indexes. For more clarity, the coordinated control action can be defined as:

$$
\begin{aligned}
M_{z, \dot{\psi}, \mathrm{comb}} & =M_{z, \dot{\psi}} \cdot \frac{\chi_{11}}{\chi_{11}+\chi_{21}}=M_{z, \dot{\psi}} \cdot \eta_{11} \\
M_{z, \beta, \text { comb }} & =M_{z, \beta} \cdot \frac{\chi_{12}}{\chi_{12}+\chi_{22}}=M_{z, \beta} \cdot \eta_{12} \\
\delta_{r, \dot{\psi}, \mathrm{comb} b} & =\delta_{r, \dot{\psi}} \cdot \frac{\chi_{21}}{\chi_{11}+\chi_{21}}=\delta_{r, \dot{\psi}} \cdot \eta_{21} \\
\delta_{r, \beta, \mathrm{comb} b} & =\delta_{r, \beta} \cdot \frac{\chi_{22}}{\chi_{12}+\chi_{22}}=\delta_{r, \beta} \cdot \eta_{22}
\end{aligned}
$$

where on the left side the obtained control actions are the combined ones while on the right side the control actions provided by the PIs controllers are present. $\eta$ are the efficiency factors that weights the controller action in combined control which are obtained by weighting the performance indexes as in equation (18).

Once the controller yawing moment $M_{z}$ and rear steering angle $\delta_{r}$ have been generated they have to be applied by actuators. The rear steering angle is requested to the RWS actuator. Instead, the rear yaw moment $M_{z}$ needs to be generated by the differentiating the driving/braking torques on the rear axis. The torque allocation block sums $M_{z}$ required torques to the driving/braking torque required by the driver. Finally an antiskid block limits the torques in order to avoid excessive tyre slips.

To have an estimation of the sideslip angle (which is not measured on commercial passenger cars) the Extended Kalman Filter (EKF) proposed in [10] is then implemented and used by the controller.

\subsection{Control references}

The control targets are the reference yaw-rate and sideslip angle. For what concerns the yaw-rate, it is related to the steering angle imposed by the driver and the understeering behavior of the vehicle.

For what concerns yaw rate reference $\dot{\psi}_{\mathrm{r}}$ it is calculated according to front steering angle $\delta_{f}$. Considering kinematic steering, accounting also for understeer coefficient $K_{U S}$, the yaw rate, in the linear region, as function of steering angle reads

$$
\dot{\psi}_{\operatorname{lin}}=\frac{v}{l\left(1+K_{U S} v^{2}\right)} \delta=\Psi \delta_{f}
$$

In order to account for maximum achievable yaw rate due to friction limit $\dot{\psi} \leq \mu g / v$ and to avoid discontinuity in reference equation yaw rate reference thus reads

$$
\dot{\psi}_{\mathrm{ref}}= \begin{cases}\dot{\psi}_{\mathrm{lin}} & |\delta| \leq \delta_{1} \\ \dot{\psi}_{1}+\left(\dot{\psi}_{\max }-\dot{\psi}_{1}\right)\left(1-e^{-\frac{\Psi\left(|\delta|-\delta_{1}\right)}{\left(\dot{\psi}_{\max }-\psi_{1}\right)}}\right) & |\delta|>\delta_{1}\end{cases}
$$

Transition point from linear to non-linear behavior $\left(\delta_{1}, \dot{\psi}_{1}\right)$ can be chosen by controller designer; it must be considered that $\delta_{1}$ and $\dot{\psi}_{1}$ are related by equation (19): $\dot{\psi}_{1}=\Psi \delta_{1}$.

For what concerns the sideslip angle it is defined such that the $\beta$ limitation is applied only if it becomes larger than the maximum admissible level and with opposite sign 
with respect to the yaw-rate (see [10]). This is made because imposing the minimization of the sideslip angle leads on the decrease of the vehicle performances since it works generally in conflicts to the yaw rate reference. Reference sideslip angle is defined as:

$$
\beta_{\text {ref }}= \begin{cases}\beta & |\beta| \leqslant \beta_{t h} \\ \beta\left[1-f_{w}\left(v, \beta, \delta_{f}\right)\right]+\beta_{t h} f_{w}\left(v, \beta, \delta_{f}\right) & |\beta|>\beta_{t h}\end{cases}
$$

where $\beta_{t h}$ is the sideslip angle threshold at which the control is activated and $f_{w}\left(v, \beta, \delta_{f}\right)$ is a weighting function introduced to smooth the passage between uncontrolled to controlled vehicle and to weight the $\beta$-control as function of the speed.

\subsection{PIs controllers and coordination}

Tracking of yaw rate and sideslip angle references is achieved by means of gain scheduled PI regulators. The generic control action (u) is thus given by:

$$
u(t)=K_{P} e(t)+K_{I} \int_{0}^{t} e(\tau), d \tau
$$

where $K_{P}$ and $K_{I}$ are respectively the proportional and the integral coefficients and $e(t)$ is the tracking error. Proportional and integral gains are scheduled to maintain sufficient bandwidth and constant damping coefficient of the simplified single-track vehicle transfer function in the Laplace domain eq. (23),

$$
\begin{aligned}
\frac{\dot{\psi}}{M_{z}} & =\frac{m v^{2} s+v\left(K_{\mathrm{f}}+K_{\mathrm{r}}\right)}{m v^{2} J_{z} s^{2}+s v\left(m\left(K_{\mathrm{f}} l_{\mathrm{f}}^{2}+K_{\mathrm{r}} l_{\mathrm{r}}^{2}\right)+J_{z}\left(K_{\mathrm{f}}+K_{\mathrm{r}}\right)\right)+\left(4 K_{\mathrm{f}} K_{\mathrm{r}} l_{\mathrm{r}}^{2}-v^{2} m\left(K_{\mathrm{f}} l_{\mathrm{f}}-K_{\mathrm{r}} l_{\mathrm{r}}\right)\right)} \\
\frac{\beta}{M_{z}} & =\frac{-\left(K_{\mathrm{f}} l_{\mathrm{f}}-K_{\mathrm{r}} l_{\mathrm{r}}+m v^{2}\right)}{m v^{2} J_{z} s^{2}+s v\left(m\left(K_{\mathrm{f}} l_{\mathrm{f}}^{2}+K_{\mathrm{r}} l_{\mathrm{r}}^{2}\right)+J_{z}\left(K_{\mathrm{f}}+K_{\mathrm{r}}\right)\right)+\left(4 K_{\mathrm{f}} K_{\mathrm{r}} l_{\mathrm{r}}^{2}-v^{2} m\left(K_{\mathrm{f}} l_{\mathrm{f}}-K_{\mathrm{r}} l_{\mathrm{r}}\right)\right)} \\
\frac{\dot{\psi}}{\delta_{r}} & =\frac{-m J_{z} v^{2} K_{\mathrm{r}}\left(K_{\mathrm{f}} l_{\mathrm{f}}+K_{\mathrm{f}} l_{\mathrm{r}}+l_{\mathrm{r}} v m s\right)}{m v^{2} J_{z} s^{2}+s v\left(m\left(K_{\mathrm{f}} l_{\mathrm{f}}^{2}+K_{\mathrm{r}} l_{\mathrm{r}}^{2}\right)+J_{z}\left(K_{\mathrm{f}}+K_{\mathrm{r}}\right)\right)+\left(4 K_{\mathrm{f}} K_{\mathrm{r}} l_{\mathrm{r}}^{2}-v^{2} m\left(K_{\mathrm{f}} l_{\mathrm{f}}-K_{\mathrm{r}} l_{\mathrm{r}}\right)\right)} \\
\frac{\beta}{\delta_{r}} & =\frac{J_{z} v s+\left(K_{\mathrm{r}} K_{\mathrm{f}} l_{\mathrm{f}}^{2}+K_{\mathrm{f}} K_{\mathrm{r}} l_{\mathrm{f}} l_{\mathrm{r}}+l_{\mathrm{r}} K_{\mathrm{r}} m v^{2}\right)}{m v^{2} J_{z} s^{2}+s v\left(m\left(K_{\mathrm{f}} l_{\mathrm{f}}^{2}+K_{\mathrm{r}} l_{\mathrm{r}}^{2}\right)+J_{z}\left(K_{\mathrm{f}}+K_{\mathrm{r}}\right)\right)+\left(4 K_{\mathrm{f}} K_{\mathrm{r}} l_{\mathrm{r}}^{2}-v^{2} m\left(K_{\mathrm{f}} l_{\mathrm{f}}-K_{\mathrm{r}} l_{\mathrm{r}}\right)\right)}
\end{aligned}
$$

where $s$ is the Laplace coordinate. They describe the linearization of the single track model equations where $K_{f}$ and $K_{r}$ are the front and rear axle cornering stiffness evaluated at the equilibrium sideslip angle. Figure 7 reports the value of the poles of the closed-loop transfer functions for increasing values of proportional gain (i.e. the root locus). The final set of gains is reported in Table 2 where the values of proportional and integral gains associated to each PI is reported for three different vehicle speed.

Moreover, when considering the actuation bandwidth, the time constant of the two actuation systems are quite different as reported in Table 1, but it has to be considered that TV operates on the longitudinal tire forces while RWS system on the lateral ones. The relaxation length in lateral direction is higher than the longitudinal one. The actuation time constant and the tire relaxation lengths provide a difference in the effective time response of the two controllers. For this reason, aiming at a good response of the rear steering system both in transient and steady state conditions, the RWS PIs bandwidth has been limited with respect to the one of TV. 

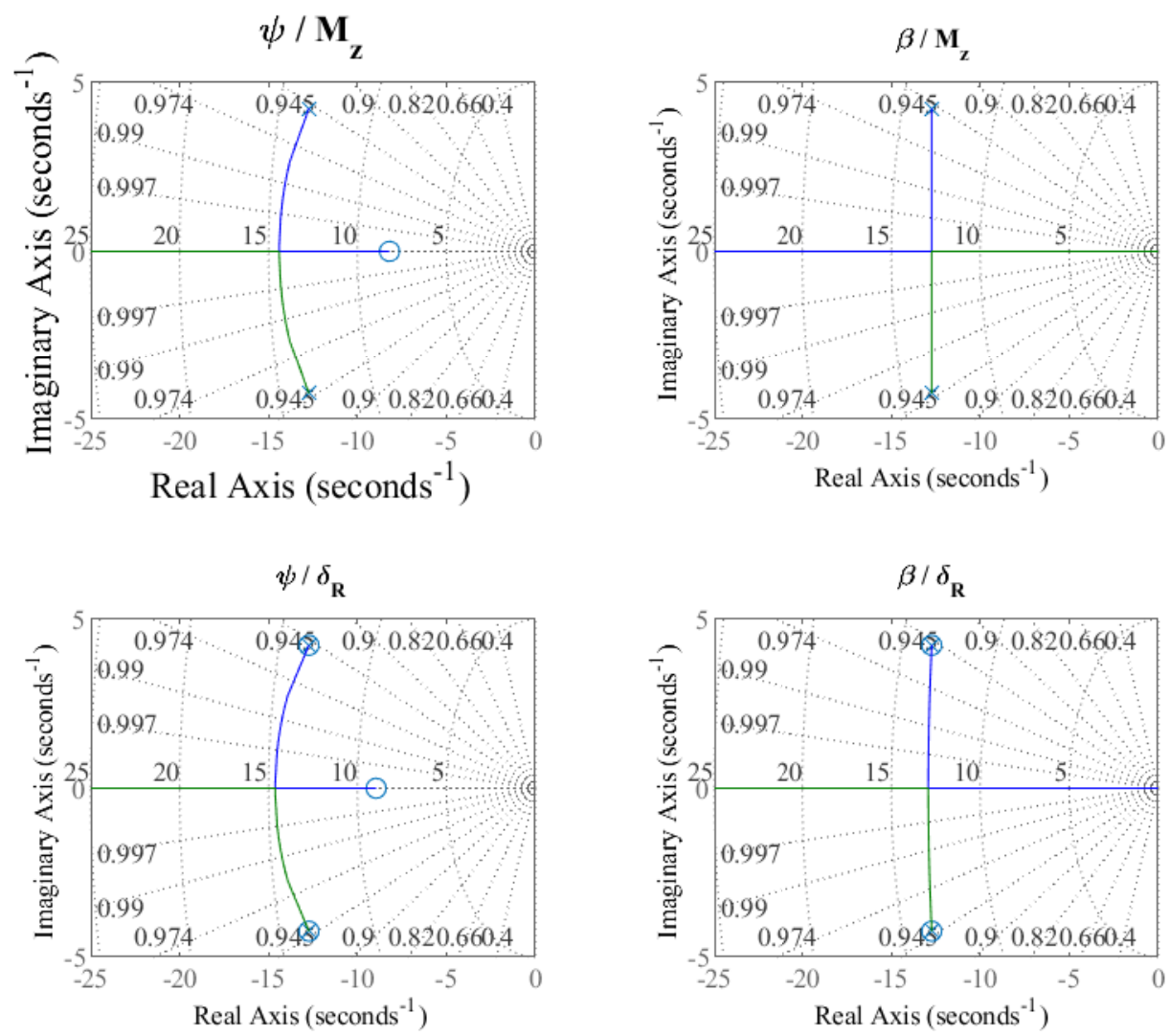

Figure 7. Root locus for system transfer functions at $70 \mathrm{~km} / \mathrm{h}$.

Table 2. Proportional and integral gains of PIs as function of vehicle speed.

\begin{tabular}{lrrr}
\hline$v[\mathrm{~m} / \mathrm{s}]$ & 10 & 15 & 20 \\
\hline $\mathrm{kP} 11$ & $2.76 \mathrm{E}+04$ & $1.84 \mathrm{E}+04$ & $1.38 \mathrm{E}+04$ \\
$\mathrm{kI} \mathrm{11}$ & $5.52 \mathrm{E}+01$ & $3.68 \mathrm{E}+01$ & $2.76 \mathrm{E}+01$ \\
$\mathrm{kP} 12$ & $2.76 \mathrm{E}+03$ & $1.84 \mathrm{E}+03$ & $1.38 \mathrm{E}+03$ \\
$\mathrm{kI} \mathrm{12}$ & $5.52 \mathrm{E}+00$ & $3.68 \mathrm{E}+00$ & $2.76 \mathrm{E}+00$ \\
$\mathrm{kP} \mathrm{21}$ & $3.00 \mathrm{E}-01$ & $2.00 \mathrm{E}-01$ & $1.50 \mathrm{E}-01$ \\
$\mathrm{kI} \mathrm{21}$ & $6.00 \mathrm{E}-04$ & $4.00 \mathrm{E}-04$ & $3.00 \mathrm{E}-04$ \\
$\mathrm{kP} \mathrm{22}$ & $-3.00 \mathrm{E}-01$ & $-2.00 \mathrm{E}-01$ & $-1.50 \mathrm{E}-01$ \\
$\mathrm{kI} \mathrm{22}$ & $-6.00 \mathrm{E}-04$ & $-4.00 \mathrm{E}-04$ & $-3.00 \mathrm{E}-04$ \\
\hline
\end{tabular}


Since for high lateral acceleration a trade-off between the reference yaw-rate and the limitation of the vehicle sideslip angle has been pointed out, an anti wind-up procedure is used to reset the integral parts of the controllers when the control actions $\left(u_{\dot{\psi}}\right.$ and $u_{\beta}$ ) point in opposite direction, i.e.

$$
u_{\dot{\psi}} u_{\beta}<0
$$

where $u$ is the generic control action $M_{z}$ or $\delta_{r}$. This permits to avoid bad effects related to the build-up of the control integral part. Moreover, to improve stability of the system, if the sideslip angle exceeds a certain threshold value the yaw-rate control is deactivated permitting the maintenance of vehicle stability even if, in doing so, performances slightly worsen.

\subsection{Torques allocation}

The allocation procedure superimposes the longitudinal force required by the driver, (which is equally partitioned between the rear wheels) to the longitudinal forces required to generate the controller yaw moment $M_{z}$. Since the total required longitudinal force, i.e. the motor torque, can be unfeasible due to the motor limitation, the fulfillment of driver's demand has been treated as soft constraint. $M_{z}$ is limited in the region between $M z_{+}$(maximum clockwise yaw moment) and $M z_{-}$(maximum counter-clockwise yaw moment) which are defined as follows

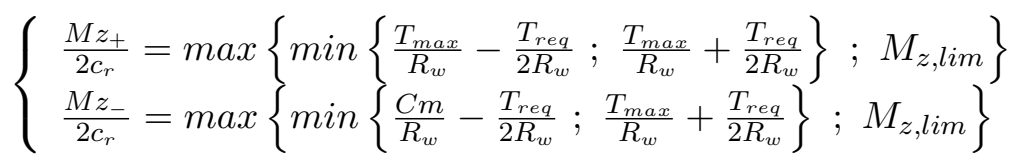

$c_{r}$ is the rear semi-track, $T_{\max }$ is the maximum motor torque, $R_{w}$ is the rear tires rolling radius, $T_{r e q}$ is the overall motors torque required by the driver and $M z_{\text {lim }}$ the minimum yaw moment accepted to satisfy the driver requirements.

\section{Simulation and results}

In this section, simulation results are reported. In particular, several maneuvers have been simulated to evaluate the steady-state and transient behavior of both the passive and the controlled vehicle. Also the coupling with the driver has been evaluated trough closed-loop maneuvers.

In the following only the most significant maneuvers are reported:

- steering pad constant radius: steady-state closed-loop maneuver, steer is actuated by the driver model to follow a fixed radius curve progressively increasing speed;

- step-steer maneuver: transient open-loop maneuver in which speed is maintained and a fast variation of the front steering angle is applied;

- double lane change: transient closed-loop maneuver with a reference trajectory;

Maneuvers are simulated in a Simulink-MATLAB environment. The vehicle model is a 14 degrees of freedom vehicle model (see Figure 8):

- 3 chassis displacements ( $x, y$ and $z$ );

- 3 chassis rotation (yaw, pitch and roll); 


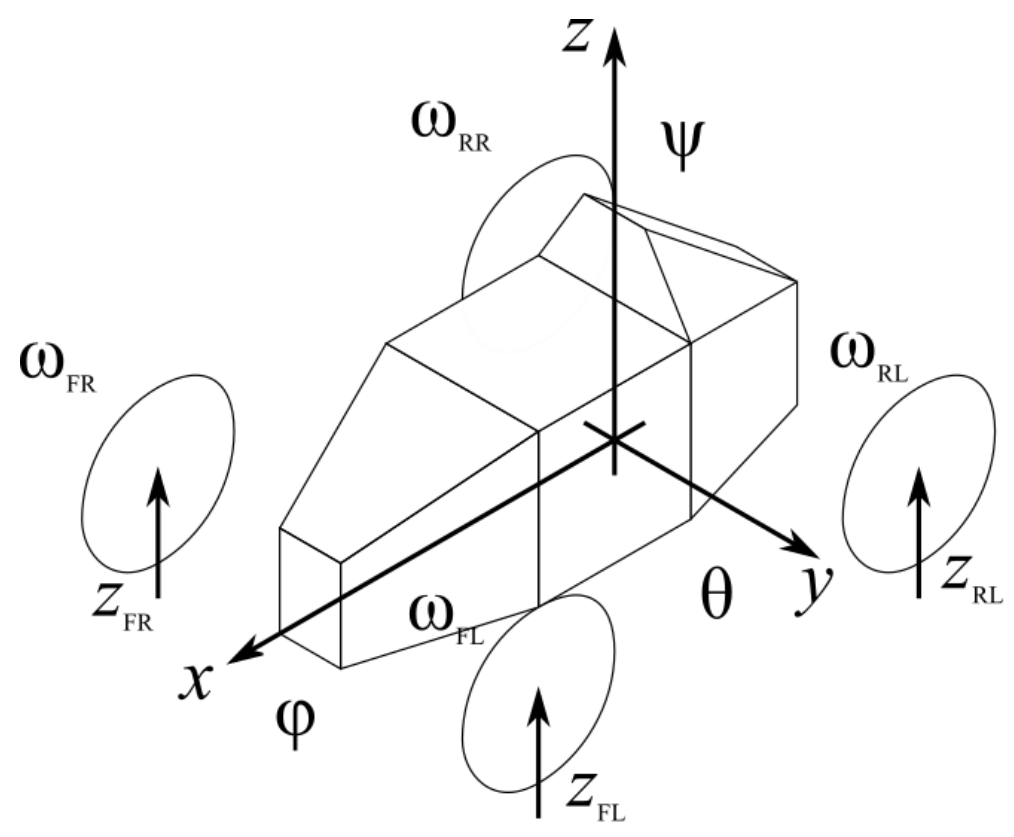

Figure 8. 14 dofs vehicle model

- 4 wheel angular speeds;

- 4 unsprung masses vertical displacements.

The tire characteristic is described by combined slip MF-Tyre model while actuators steady-state characteristics and bandwidth [10]. Both open-loop and closed-loop maneuvers are performed. The closed-loop maneuvers are simulated with a driver model, as reported in [17], able to follow the desired trajectory.

The steer angle allowing to follow a given trajectory is determined based on two error functions

$$
\delta=k_{1} e_{1}+k_{2} e_{2}
$$

where $k_{1}$ and $k_{2}$ are the driver model gains, while the error functions are defined as

$$
\begin{aligned}
& e_{1}=\psi_{r e f}(s)-\psi(s) \\
& e_{2}=\left[P_{x, r e f}\left(s+l_{s}\right)-P_{G, x}(s)\right] \cos \left(\psi_{r e f}(s)\right)+\left[P_{y, r e f}\left(s+l_{s}\right)-P_{G, y}(s)\right] \sin \left(\psi_{r e f}(s)\right)
\end{aligned}
$$

The error function $e_{1}$ represents the difference between the angle of the tangent to the desired path $\left(\psi_{r e f}\right)$ and the vehicle yaw angle (i.e. it represents the error on the vehicle orientation), while $e_{2}$ is the error between the actual position of the center of gravity $(\operatorname{cog})$ of the vehicle (PGx, PGy) and its corresponding position along the reference path. $s$ is the reference trajectory curvilinear abscissa and $l_{s}$ a preview length which is a function of vehicle speed.

\subsection{Compared control strategies}

To validate the performances of the coordinated control, simulations are performed by comparing the passive vehicle with three different controlled vehicles. The following 


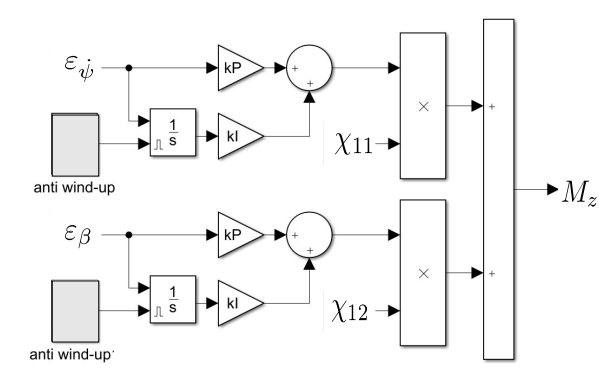

a) Torque Vectoring

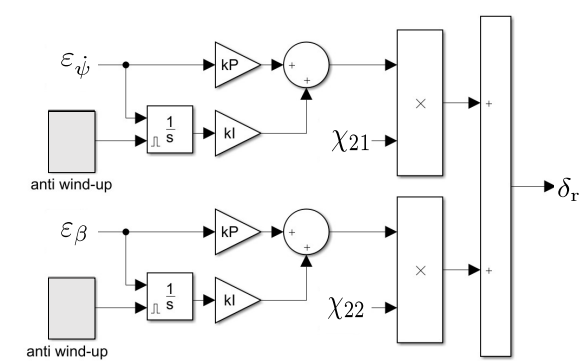

b) Rear Wheel Steering

Figure 9. Scheme of the TV (a) and RWS (b) controllers reported in simulation results.

vehicles are compared:

passive passive vehicle;

TV Torque Vectoring controlled vehicle is a control based on the same architecture of the combined control. Both sideslip and yaw-rate are controlled by the TV. The scheme of this controller is reported in Figure 9.a;

RWS Rear Wheel Steering controlled vehicle is a control based on pure RWS actuation system. The scheme of this controller is reported in Figure 9.b;

combined combined multi-actuated vehicle presents both TV and RWS that work together adopting the control strategy described in section 4 .

Comparing results obtained by the different control systems, some results can be highlighted looking at the advantages and disadvantages of each actuation system with respect to a multi-actuated system and a multi-actuated system with some forms of coordination among them.

\subsection{Steering pad constant radius}

The steering pad constant radius maneuver is a closed-loop maneuver in which the vehicle is driven in a circular path of constant radius and the speed is slowly increased. This maneuver allows to evaluate the steady-state performance of the vehicle. In particular, the understeering coefficient can be evaluated together with the maximum achievable lateral acceleration.

Table 3. Steering pad constant radius on high friction. Steering wheel angle $\delta_{f} / \delta_{0}$ as function of lateral acceleration.

\begin{tabular}{llllllllllll}
\hline$a_{y}\left[\mathrm{~m} / \mathrm{s}^{2}\right]$ & 2 & 3 & 4 & 5 & 6 & 7 & 8 & 9 & 10 & 11 & 11.6 \\
\hline passive & 1.00 & 1.01 & 1.02 & 1.03 & 1.05 & 1.06 & 1.08 & 1.10 & 1.13 & 1.2 & - \\
TV & 1.00 & 1.00 & 1.00 & 1.00 & 1.01 & 1.01 & 1.02 & 1.03 & 1.30 & 2.20 & - \\
RWS & 1.00 & 1.00 & 1.01 & 1.02 & 1.02 & 1.02 & 1.04 & 1.06 & 1.31 & 1.7 & - \\
combined & 1.00 & 1.00 & 1.00 & 1.00 & 1.00 & 1.00 & 1.01 & 1.01 & 1.13 & 1.7 & 3.50 \\
\hline
\end{tabular}

Results of the steering pad are shown in Figure 10 and in Table 3 where steering angle ratio $\left(\delta_{f} / \delta_{0}\right)$ and sideslip angle $(\beta)$ are represented as function of lateral acceleration $a_{y}$. As noticeable, the passive vehicle presents an increasing under-steering 

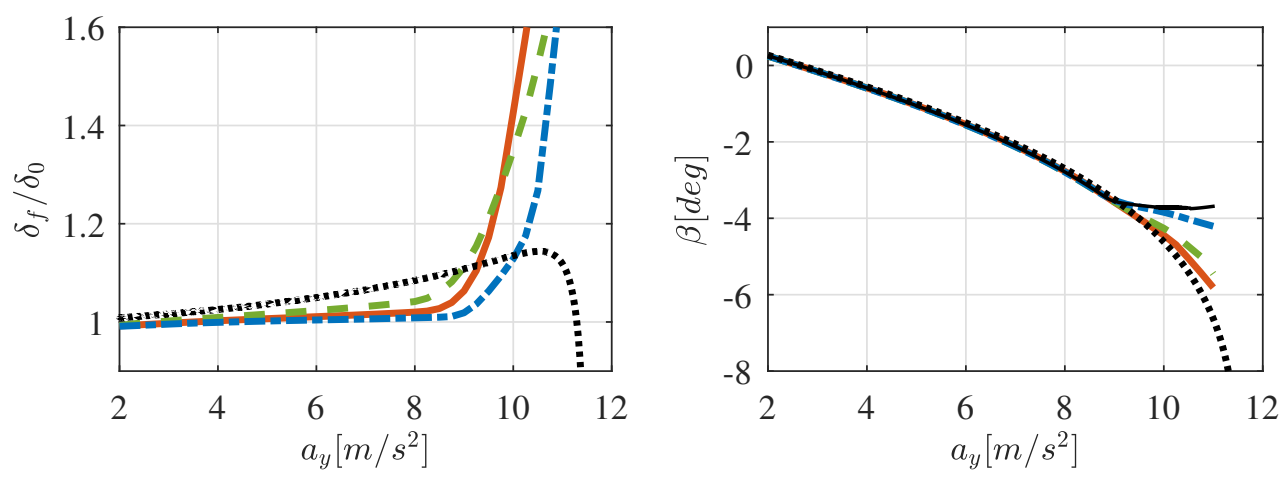

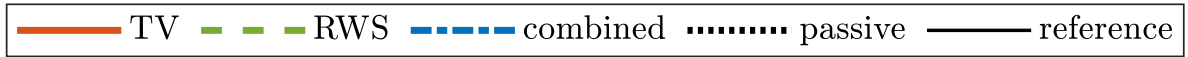
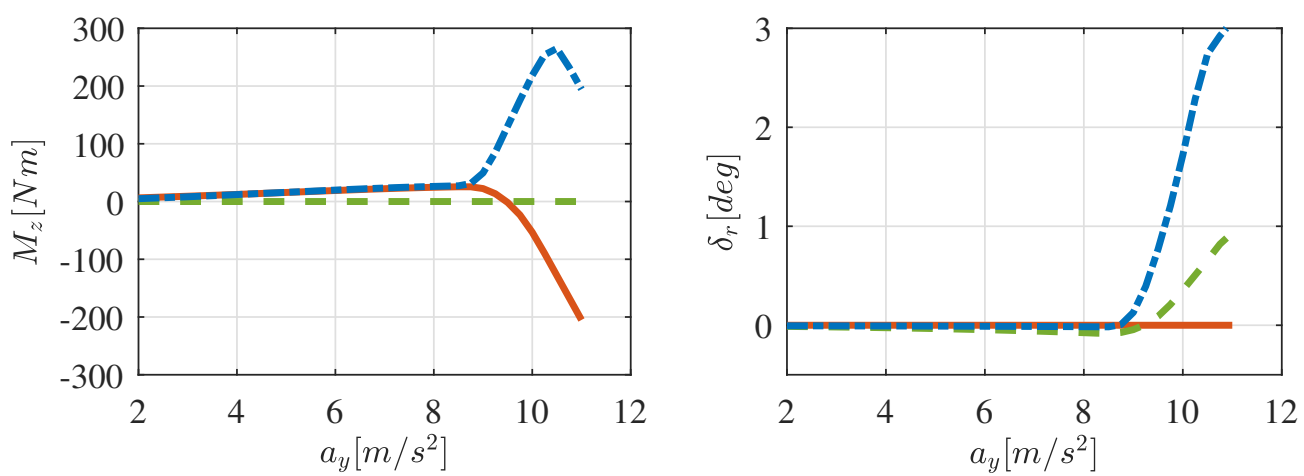

Figure 10. Steering pad constant radius $(50 \mathrm{~m})$ maneuver on high friction road $\mu=1$. Comparison between controlled and passive vehicles.

behavior and the stability of the vehicle is granted only by the driver model skills up to the friction limit where the passive vehicle becomes fully uncontrollable. On the other hand, all the controlled vehicles present a more neutral behavior until the sideslip angle exceeds the threshold of $3.5^{\circ}\left(\beta_{t h}\right)$, above which the sideslip angle limitation kicks in. However, combined control is the one that better succeed in making the vehicle neutral up to higher lateral accelerations. For lateral accelerations close to the friction limit, the limitation of sideslip angle produces an understeering behavior. This progressive trend allows the driver to identify the approching of the limit condition. Moreover, the combined control highlights the capability to reach higher maximum lateral acceleration with respect to the others. ${ }^{1}$

RWS is instead the worsen and is the one that most suffer the tracking of the two conflicting references. Less improvements are shown with respect to combined control in keeping the vehicle close to neutral behavior (steering increases more than TV and combined). This reflects also on side slip angle tracking where, at high lateral acceleration, only combined control, thanks to the addition of $\mathrm{TV}$, is capable in remaining close to side slip angle reference.

\footnotetext{
${ }^{1}$ It should be noticed that the, even if the friction coefficient is 1 , the lateral acceleration is higher than $g$ because, according to eq. 7 , the peak of lateral force depends also on $\left(p_{1}+p_{2} d f_{z}\right)$ which, for the considered tire, is higher than 1.
} 
TV places in between. This is a first proof that having a multi-actuated vehicle leads to some advantages on the vehicle dynamics. This is confirmed by looking at Figure 10 where the control action $\left(M_{z}\right.$ and $\left.\delta_{r}\right)$ are reported as function of $a_{y}$. It is possible to see that up to $9 \mathrm{~m} / \mathrm{s}^{2}$ all the controls are focused only on maintaining the vehicle neutral behavior by applying a positive rear yaw moment that neutralizes the vehicle under-steering behavior or by a negative rear steering angle that generates an equivalent effect. Beyond $9 \mathrm{~m} / \mathrm{s}^{2}$, the vehicle reaches the maximum admissible value of sideslip angle, RWS and Combined control both increase the rear steering angle (in the same direction of the front one) to keep the same value of lateral acceleration limiting the increase of sideslip angle. On the other hand, in the same condition, TV changes the yaw moment direction (from positive to negative) to limit sideslip angle thus increasing the understeering gradient and reducing the performances in increasing the lateral acceleration. The same happens to the RWS controlled vehicle that limiting $\beta$ improves the vehicle under-steering behavior. For what regards the multi-actuated vehicles (combined control), the balance between the positive rear steer angle and the positive yaw moment provides beneficial results in maintaining a limited steer gradient and sideslip angle.

In this maneuver the combined control shows RWS saturating by reaching 3 degrees, this makes the controller to use TV for both controlling yaw rate and sideslip angle. Since these targets are conflicting the tracking error increases but is still smaller than TV alone. Since RWS saturates, the actuated vehicle becomes single-actuated by TV. This makes TV to compromise between the two targets. To avoid excessive increase of the sideslip angle the performance in tracking yaw rate degrades in favor of vehicle safety.

\subsection{Step steer maneuver}

Step steer is an open-loop maneuver that aims to analyze the transient response of the vehicle. A fast front steering angle variation is imposed in a small amount of time and it is maintained to a sufficient amount of time to analyze the vehicle behavior. During the test, the vehicle speed is kept nearly constant by the cruise control. In this case, results are reported in the time domain.

Here the result of a step-steer maneuver at a speed of $50 \mathrm{~km} / \mathrm{h}$ and a steering wheel step of 25 degrees are reported in table 4 and Figure 11.

Table 4. Step-steer maneuver on high friction road surface at $50 \mathrm{~km} / \mathrm{h}$ with a steer wheel step of $25^{\circ}$.

\begin{tabular}{lccccc}
\hline & & passive & TV & RWS & combined \\
\hline$a_{y, s s}$ & {$\left[\mathrm{~m} / \mathrm{s}^{2}\right]$} & - & 11.14 & 11.15 & 11.67 \\
$a_{y, \max }$ & {$\left[\mathrm{m} / \mathrm{s}^{2}\right]$} & - & 11.21 & 11.70 & 11.72 \\
$t_{a_{y}}$ & {$[\mathrm{~s}]$} & - & 0.33 & 0.36 & 0.35 \\
$\dot{\psi}_{s s}$ & {$[\mathrm{deg} / \mathrm{s}]$} & - & 46.66 & 46.74 & 48.98 \\
$\dot{\psi}_{\max }$ & {$[\mathrm{deg} / \mathrm{s}]$} & - & 53.55 & 55.60 & 53.59 \\
$t_{\dot{\psi}}$ & {$[\mathrm{s}]$} & - & 0.22 & 0.24 & 0.22 \\
$\beta_{s s}$ & {$[\mathrm{deg}]$} & - & -4.36 & -4.29 & -4.30 \\
$\beta_{\max }$ & {$[\mathrm{deg}]$} & - & -4.65 & -5.59 & -4.46 \\
$t_{\beta}$ & {$[\mathrm{s}]$} & - & 0.48 & 0.46 & 0.44
\end{tabular}

$\star_{s s}$ refer to value assumed by the $\star$ quantity at the steady state condition. $\star_{\max }$ refer to the maximum value (in module) assumed by $\star . t_{\star}$ indicate the times between the steer step starting time $\left(t_{0}=0.5 \mathrm{~s}\right)$ and the time at which $\star$ assume a value equal to the $90 \%$ of the steady state one. Value - is reported if the vehicle is not stable. 
By looking at Figure 11 it can be noticed that the passive vehicle cannot accomplish the maneuver. All the controlled vehicles are instead stable but with significant differences.

Looking at the response of sideslip angle, yaw rate, accelerations and speed, combined control shows the smoothest, less oscillating, behavior. Parallel and TV controlled vehicles present an overshoot and then reduced oscillations. RWS controlled vehicle presents the most oscillating behavior. This is also highlighted by the time history of $\delta_{r}$.

Moreover, multi-actuated control denote a higher maximum lateral acceleration generated in a comparable $t_{a_{y}}$ with respect to the single-actuated one. Specifically, combined control presents a $a_{y, \max }$ which is $4.8 \%$ higher than the TV one. As already reported, the response of the RWS is slower than the one of the others configurations. This generates a high number of oscillations. With regards to the sideslip angle, all the controls succeed in reaching comparable steady-state values in similar time windows with the minimum overshoot reached by the combined one.

Analyzing $M_{z}$ and $\delta_{r}$, it is possible to notice that until $\beta_{t h}$ is reached, a yaw moment helping the vehicle to enter into the turn (same direction of $\delta_{f}$ ) and $\delta_{r}$ in the opposite direction of $\delta_{f}$ are generated to decrease the vehicle under-steering behavior. Contrarily, when the control is on the sideslip angle, opposite control actions are needed. This provides a higher loss of performance for the single-actuated control with respect to the multi-actuated one. Moreover, the rear steering angle reaches easily the saturation condition decreasing the capability of RWS and parallel control to maintain vehicle stability. Furthermore, analyzing the combined control actions, when the sideslip angle presents a high gradient, it is principally managed by the TV that is less effective but faster. Then once the sideslip angle trend has changed, this task is assumed by the RWS and the yaw-moment is increased to reduce the vehicle under-steering behavior.

\subsection{Double lane change maneuver}

The double lane change is a closed-loop maneuver. It consists in following a trajectory composed by a straight part, a lateral change to reach a parallel straight line and a then another change in lateral position to return on the original straight line. During the test, the maximum entrance speed that guarantees to remain inside the track boundaries is searched.

The simulation is performed by assuming a low friction coefficient of 0.5 to simulate low adherence conditions.

The results of the maneuver are reported in Figure 12. As it can be noticed from the initial speed value $\left(v_{x}\right.$ at $\left.t=0 \mathrm{~s}\right)$, an improvement of $6.5 \%$ of the entrance velocity is obtained by controlled vehicles with respect to passive vehicle.

Results for controlled vehicles are comparable where in general a reduction of the oscillation can be noticed. Moreover, a lower driver workload and lower maximum lateral acceleration, yaw-rate and sideslip angle characterize the controlled vehicles.

RWS is the worsen in terms of sideslip angle and yaw rate peaks at about 6 seconds.

Looking at applied yaw moment, combined control shows similar yaw moment $M_{z}$ with respect to TV but with smaller peak values. The same trend can be observed comparing the use of rear steering by RWS and combined control. The shape of $\delta_{r}$ is similar but combined control achieves better results with smaller peak values of $\delta_{r}$. 


\subsection{Simulation results conclusions}

Summarizing the obtained results, following considerations can be drawn. From steadystate simulation, steering pad constant radius of Figure 10, higher maximum lateral acceleration with limited sideslip angle can be achieved with combined control and less under-steering vehicle behavior up to the activation of the sideslip angle limitation.

Combined control performance can be better analyzed by looking at Figure 13 where the performance indexes $(\chi)$ and weighting coefficients $(\eta)$ are reported.

Regarding yaw rate (Figure 13 left side plots), for small lateral acceleration values (i.e. at the beginning of the maneuver when speed is low), the capability of RWS in increasing yaw rate is higher than TV $\left(\chi_{21+}>\chi_{11+}\right)$ thus RWS is preferred in controlling yaw rate $\left(\eta_{21}>\eta 11\right)$. Viceversa, while lateral acceleration increases (i.e. for time from 25 to 40 seconds) the capability of TV in increasing yaw rate is higher than RWS $\left(\chi_{11+}>\chi_{21+}\right)$ and TV control has higher weight with respect to RWS $\left(\eta_{11}>\eta 21\right)$. At the end of the simulation (time larger than $40 \mathrm{~s}$ ) both the performances of the two controllers are reducing mainly due to actuator saturation (see Figure 10) but TV is still preferable $\left(\eta_{11}>\eta 21\right)$.

Talking about sideslip angle (Figure 13 right side plots), RWS shows superior capability in increasing the side slip angle as required by the reference. However, its performance index is decreasing in time due to physical behavior of the vehicle and actuator saturation.

Figure 13 reports performance indexes $(\chi)$ and weighting coefficients $(\eta)$ for step steer maneuver.

Regarding TV capability in modifying yaw rate it can be noticed that, after the steer step, when the yaw rate approaches the maximum value allowed by friction $\left(\dot{\psi}_{\max }=\mu g / v \approx 48 \mathrm{deg} / \mathrm{s}\right.$, with $\mu=1.2, g=9.81 \mathrm{~m} / \mathrm{s}^{2}$ and $\left.v=50 \mathrm{~km} / \mathrm{h}\right)$, TV is able to increase the yaw rate with respect to passive vehicle $\left(\chi_{11+} \approx 0.7\right)$ but not to decrease yaw rate more that the passive $\left(\chi_{11-}<0.1\right)$. RWS shows smaller capability in modifying yaw rate with respect to $\mathrm{TV}$, but its performances are similar in both increasing or decreasing yaw rate $\left(\chi_{21+} \approx 0.15\right.$ and $\left.\chi_{21-} \approx 0.1\right)$. It has to be noticed that, increasing yaw rate beyond $\dot{\psi}_{\max }$ is possible but generally this means to reach a spinning condition with loss of vehicle controllability. Controllers performances in tracking sideslip angle can be seen from right diagrams of Figure 13. TV can further reduce sideslip angle $\left(\chi_{12-} \approx 0.25\right)$, which is negative, but it presents poor capability in increasing it with respect to passive natural behavior $\left(\chi_{12+} \approx 0\right)$, i.e. in reducing it in absolute value. RWS instead shows both capability in increasing and reducing sideslip angle with respect to passive $\left(\chi_{22+} \approx 0.05\right.$ while $\left.\chi_{22-} \approx 0.1\right)$. Finally, due to previous considerations, when the vehicle reaches steady state conditions, i.e. for time grater than $1.5 \mathrm{~s}$, the controller is preferring TV to track yaw rate reference $\left(\eta_{11}>>\eta_{21}\right)$ while it is preferring RWS to track sideslip angle $\left(\eta_{22}>>\eta_{12}\right)$. During transient, i.e. when time is between 0.5 and 1.5 seconds, TV is preferred also in tracking sideslip angle.

Regarding actuator promptness, the step steer maneuver (Figure 11), as well as double lane change (Figure 12), show poorer performances of RWS with respect to TV and combined control. Although RWS can stabilize the vehicle, step steer results show higher oscillation of RWS vehicle with respect to other controlled vehicles.

From double lane change (Figure 12), less driver workload to follow the same reference trajectory (front steer angle is smaller) for all the controlled vehicles, furthermore, the entrance speed of controller vehicle is about $5 \%$ higher than passive vehicle to successfully complete the maneuver. 
Performance indexes and weighting coefficients for double lane change maneuver are reported in Figure 15. It can be noticed that, TV and RWS shows comparable performances in changing vehicle yaw rate and this reflects in an almost 45-55 distribution between TV and RWS $\left(\eta_{11} \approx 0.45\right.$ and $\left.\eta_{12} \approx 0.55\right)$. For what concerns sideslip angle instead, the tracking duty is almost completely demanded to RWS apart from the beginning of the first lane change, at about $1 \mathrm{~s}$, and at the end of the maneuver, at about $6 \mathrm{~s}$, when the vehicle returns to the starting lane when 20-80 distribution is found.

\section{Conclusion}

In this paper, a new lateral dynamics control logic for electric vehicles is proposed. Specifically, the attention of the work is focused on the coordination of two different actuation systems: the torque vectoring (TV), performed thanks to two electric motors that independently power the left and right rear wheels, and the rear wheels steering system (RWS).

The presented control strategy is a hierarchical control that presents three different layers. In the first layer parallel proportional-integral controllers evaluate the control action needed for each single actuator system to follow the reference yaw-rate and sideslip angle. In the second layer, the coordination procedure is carried out on the basis of some performance index maps weighting the control action and partitioning the control actions between the two actuators.

In the last layer, the electric motors torque allocation is performed to fulfill the yaw moment evaluated as TV control action and torque requested by the driver.

The performance indexes that are used to weight the control actions have been calculated according to the capability of each actuator to modify the vehicle dynamic behavior. In particular, strengths and weaknesses of the two actuation systems are studied through the use of phase portrait analysis. Synthetically, the performance indexes have been defined as the maximum variation of the sideslip angle $(\dot{\beta})$ and yaw-rate derivatives $(\ddot{\psi})$ with respect to the passive vehicle that the two actuation systems are able to independently generate for a given $\beta-\dot{\psi}$ point in the phase plane.

The performance index maps allow to identify the working area of the two actuators. In particular, RWS is found to have poorer performances in controlling yaw rate with respect to TV. Viceversa, RWS capability in controlling sideslip angle is higher than TV in particular for in tire linear region (yaw rate far from friction limit). TV capability in tracking sideslip angle increases instead when approaching the friction limit; this is probably due to combined slip condition.

To asses the effectiveness of combined control it was compared with single-actuated vehicles: one with TV, the other with RWS only. Both steady-state and transient maneuvers are considered and for each, both open-loop and closed-loop maneuvers are tested.

From simulation results, the new combined control method has demonstrated to improve the vehicle performance in all the conditions with respect not only to the passive vehicle but also to the other single input control methodologies.

From steady-state simulation, steering pad constant radius of Figure 10, higher maximum lateral acceleration with limited sideslip angle can be achieved with combined control and less under-steering vehicle behavior up to the activation of the sideslip angle limitation.

Regarding actuator promptness, the step steer maneuver, as well as double lane 
change, show poorer performances of RWS with respect to TV and combined control. Although RWS can stabilize the vehicle, step steer results show higher oscillation of RWS vehicle with respect to other controlled vehicles.

From double lane change, less driver workload to follow the same reference trajectory (front steer angle is smaller) for all the controlled vehicles, furthermore, the entrance speed of controller vehicle is about $5 \%$ higher than passive vehicle to successfully complete the maneuver.

\section{References}

[1] Park J, Jeong H, Jang IG, et al. Torque distribution algorithm for an independently driven electric vehicle using a fuzzy control method. Energies. 2015;8(8):8537-8561.

[2] Jin L, Liu Y. Study on adaptive slid mode controller for improving handling stability of motorized electric vehicles. Mathematical Problems in Engineering. 2014;2014.

[3] De Novellis L, Sorniotti A, Gruber P, et al. Torque vectoring for electric vehicles with individually controlled motors: State-of-the-art and future developments. World Electric Vehicle Journal. 2012;5(2):617-628.

[4] Vignati M, Sabbioni E, Cheli F. Advanced driver assistance control for drifting with torque vectoring. Proceedings of the 14th International Symposium on Advanced Vehicle Control (AVEC' 18). 2018;

[5] Vignati M, Sabbioni E, Tarsitano D, et al. Electric powertrain layouts analysis for controlling vehicle lateral dynamics with torque vectoring. In: 2017 International Conference of Electrical and Electronic Technologies for Automotive; jun. IEEE; 2017. p. 1-5. Available from: http://ieeexplore.ieee.org/document/7993204/.

[6] De Novellis L, Sorniotti A, Gruber P, et al. Comparison of feedback control techniques for torque-vectoring control of fully electric vehicles. IEEE Transactions on Vehicular Technology. 2014;63(8):3612-3623.

[7] Sabbioni E, Vignati M, Sironi G. A torque-vectoring control logic for IWM electric vehicles. In: Proc. of 12th International Symposium on Advanced Vehicle Control (AVEC'14); 2014. p. 678-685.

[8] Vignati M, Sabbioni E. Torque Vectoring Control for different powertrain layouts of hybrid and electric vehicles. Advanced Vehicle Control AVEC16 - Proceedings of the 13th International Symposium on Advanced Vehicle Control AVEC16. 2017;:637-642.

[9] Cheli F, Melzi S, Sabbioni E, et al. Torque vectoring control of a four independent wheel drive electric vehicle. In: Proceedings of the ASME Design Engineering Technical Conference; Vol. 1; 2013.

[10] Vignati M, Sabbioni E, Tarsitano D. Torque vectoring control for IWM vehicles. International Journal of Vehicle Performance. 2016;2(3):302. Available from: http://www.inderscience.com/link.php?id $=78561$.

[11] Braghin F, Sabbioni E, Sironi G, et al. A feedback control strategy for torque-vectoring of IWM vehicles. In: Proceedings of the ASME Design Engineering Technical Conference; Vol. 3; 2014.

[12] Sawase K, Ushiroda Y, Miura T. Left-right torque vectoring technology as the core of super all wheel control (S-AWC). Mitsubishi Motors Technical Review. 2006;18:16-23.

[13] Kakalis L, Cheli F, Sabbioni E. The development of a brake based torque vectoring system for a sport vehicle performance improvement. In: 6th International Conference on Informatics in Control, Automation and Robotics (ICINCO 2009), Vol. 1 ICSO; 2009. p. 298-304.

[14] Goggia T, Sorniotti A, De Novellis L, et al. Integral sliding mode for the torque-vectoring control of fully electric vehicles: Theoretical design and experimental assessment. IEEE transactions on vehicular technology. 2015;64(5):1701-1715.

[15] Siampis E, Velenis E, Longo S. Rear wheel torque vectoring model predictive control with 
velocity regulation for electric vehicles. Vehicle System Dynamics. 2015;53(11):1555-1579.

[16] Smith EN, Velenis E, Tavernini D, et al. Effect of handling characteristics on minimum time cornering with torque vectoring. Vehicle System Dynamics. 2018;56(2):221-248.

[17] Sabbioni E, Cheli F, Vignati M, et al. Comparison of torque vectoring control strategies for a IWM vehicle. SAE International Journal of Passenger Cars-Electronic and Electrical Systems. 2014;7(2014-01-0860):565-572. Available from: http://papers.sae.org/2014-010860/.

[18] Vignati M, Sabbioni E, Cheli F. A torque vectoring control for enhancing vehicle performance in drifting. Electronics (Switzerland). 2018;7(12).

[19] Besselink I, Veldhuizen T, Nijmeijer H. Improving yaw dynamics by feedforward rear wheel steering. In: IEEE Intelligent Vehicles Symposium; 2008. p. 246-250.

[20] Canale M, Fagiano L. Comparing rear wheel steering and rear active differential approaches to vehicle yaw control. Vehicle System Dynamics. 2010;48(5):529-546.

[21] Selmanaj D, Corno M, Sename O, et al. Advantages of rear steer in LTI and LPV vehicle stability control. In: Decision and Control (CDC), 2013 IEEE 52nd Annual Conference on; IEEE; 2013. p. 3523-3528.

[22] Tchamna R, Youn I. Yaw rate and side-slip control considering vehicle longitudinal dynamics. International Journal of Automotive Technology. 2013;14(1):53-60.

[23] Di Cairano S, Tseng HE, Bernardini D, et al. Vehicle yaw stability control by coordinated active front steering and differential braking in the tire sideslip angles domain. IEEE Transactions on Control Systems Technology. 2013;21(4):1236-1248.

[24] Li B, Yu F. Design of a vehicle lateral stability control system via a fuzzy logic control approach. Proceedings of the Institution of Mechanical Engineers, Part D: Journal of Automobile Engineering. 2010;224(3):313-326.

[25] Gordon T, Howell M, Brandao F. Integrated control methodologies for road vehicles. Vehicle System Dynamics. 2003;40(1-3):157-190.

[26] Edelmann J, Plöchl M. Analysis of controllability of automobiles at steady-state cornering considering different drive concepts. The Dynamics of Vehicles on Roads and Tracks. 2018;

[27] Marino R, Scalzi S, Cinili F. PI Front Steering and PI Rear Steering Control with Tire Workload Analysis. In: Intelligent Vehicles Symposium, 2007 IEEE; IEEE; 2007. p. 799804.

[28] Marino R, Scalzi S, Cinili F. Nonlinear PI front and rear steering control in four wheel steering vehicles. Vehicle System Dynamics. 2007;45(12):1149-1168.

[29] Pacejka HB. Tyre and Vehicle Dynamics. ; 2006. Available from: http://books.google.pt/books?id=wHlkbBnu9FEC.

[30] Edelmann J, Plöchl M. Handling characteristics and stability of the steady-state powerslide motion of an automobile. Regular and Chaotic Dynamics. 2009 dec;14(6):682-692. Available from: http://link.springer.com/10.1134/S1560354709060069.

[31] Rossa F, Gobbi M, Mastinu G, et al. Bifurcation analysis of a car and driver model. Vehicle System Dynamics. 2014;52(SUPPL. 1):142-156.

[32] Bobier-Tiu CG, Beal CE, Kegelman JC, et al. Vehicle control synthesis using phase portraits of planar dynamics. Vehicle System Dynamics. 2018 jul;3114:1-20. Available from: https://www.tandfonline.com/doi/full/10.1080/00423114.2018.1502456.

[33] Xiong L, Yu Z, Wang Y, et al. Vehicle dynamics control of four in-wheel motor drive electric vehicle using gain scheduling based on tyre cornering stiffness estimation. Vehicle system dynamics. 2012;50(6):831-846.

[34] Zhao C, Xiang W, Richardson P. Vehicle lateral control and yaw stability control through differential braking. In: Industrial Electronics, 2006 IEEE International Symposium on; Vol. 1; IEEE; 2006. p. 384-389.

[35] Ren B, Chen H, Zhao H, et al. MPC-based yaw stability control in in-wheel-motored EV via active front steering and motor torque distribution. Mechatronics. 2016;38:103-114.

[36] Guo H, Hao N, Chen H. Lateral stability controller design for electrical vehicle based on active rear wheel steering. In: Intelligent Control and Automation (WCICA), 2016 12th 
World Congress on; IEEE; 2016. p. 1285-1290.

[37] Poussot-Vassal C, Sename O, Dugard L, et al. Vehicle dynamic stability improvements through gain-scheduled steering and braking control. Vehicle System Dynamics. 2011; 49(10):1597-1621.

[38] Jalali M, Khajepour A, Chen Sk, et al. Integrated stability and traction control for electric vehicles using model predictive control. Control Engineering Practice. 2016;54:256-266.

[39] Beal CE, Gerdes JC. Model predictive control for vehicle stabilization at the limits of handling. IEEE Transactions on Control Systems Technology. 2013;21(4):1258-1269.

[40] Jalali K, Uchida T, McPhee J, et al. Integrated Stability Control System for Electric Vehicles with In-wheel Motors using Soft Computing Techniques. SAE International Journal of Passenger Cars - Electronic and Electrical Systems. 2009 apr;2(1):2009-01-0435. Available from: https://www.sae.org/content/2009-01-0435/.

[41] Junmin Wang, Longoria R. Coordinated and Reconfigurable Vehicle Dynamics Control. IEEE Transactions on Control Systems Technology. 2009 may;17(3):723-732. Available from: http://ieeexplore.ieee.org/document/4806119/.

[42] Canale M, Fagiano L, Ferrara A, et al. Vehicle Yaw Control via Second-Order SlidingMode Technique. IEEE Transactions on Industrial Electronics. 2008 nov;55(11):39083916. Available from: http://ieeexplore.ieee.org/document/4602704/.

[43] Utkin V, Guldner J, Shi J. Sliding Mode Control in ElectroMechanical Systems. CRC Press; 2017. October 2013; Available from: https://www.taylorfrancis.com/books/9781420065619. 

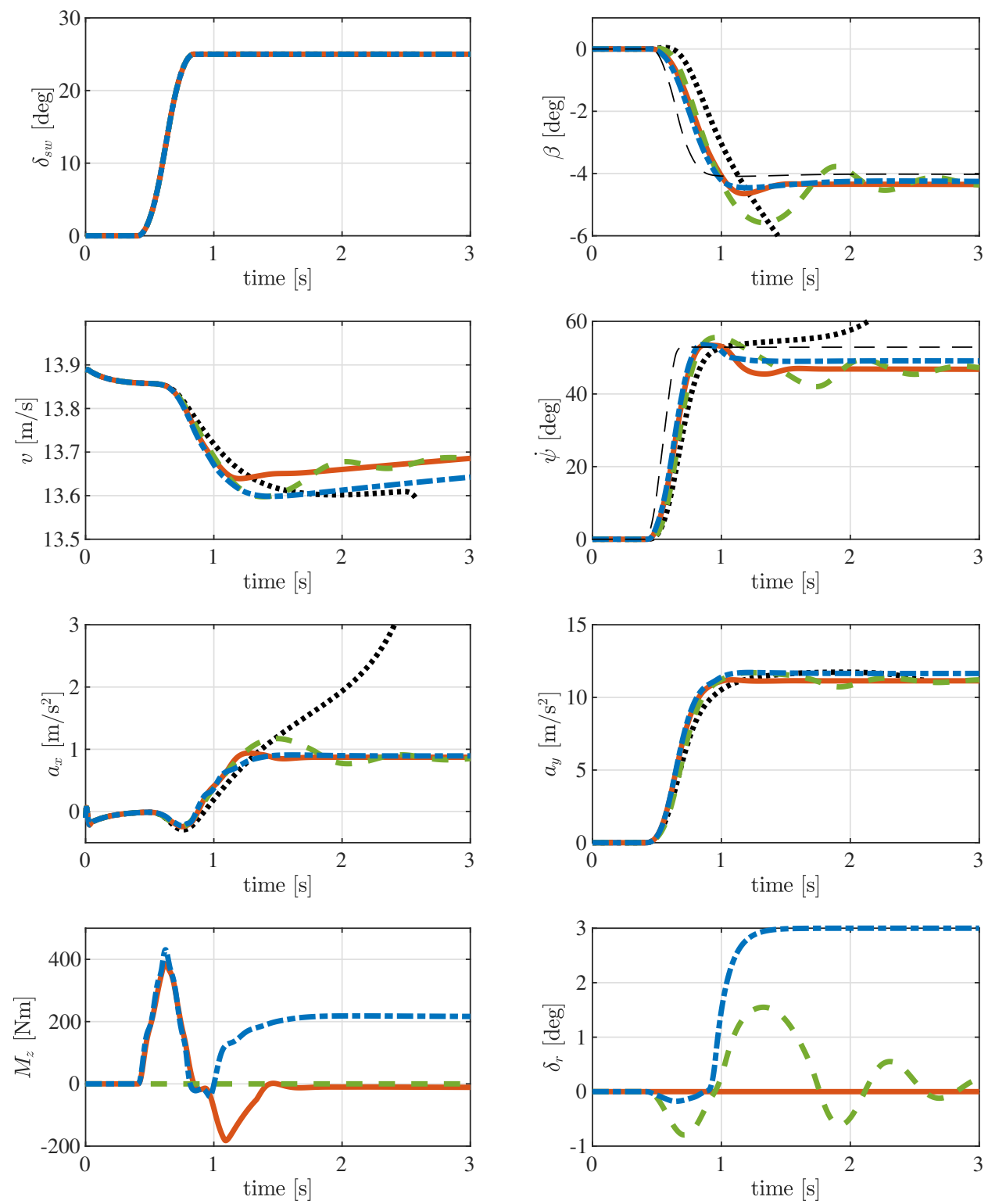

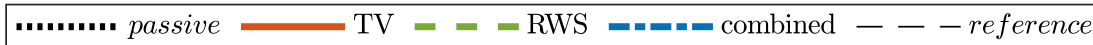

Figure 11. Simulation data of a steer step maneuver on high friction road surface: steer step of $25 d e g$ at $50 \mathrm{~km} / \mathrm{h}$. 

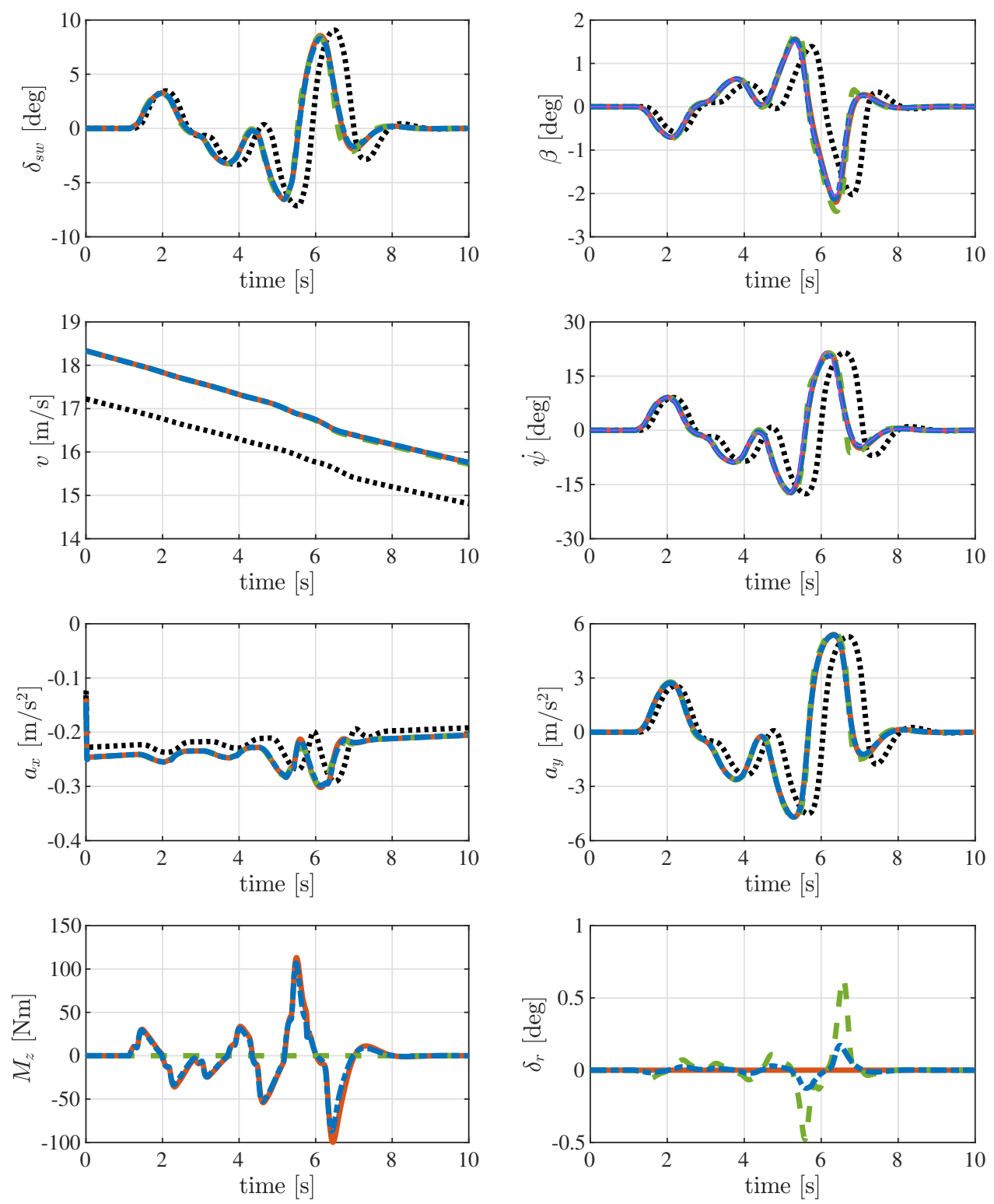

.......... passive 2 TV $-2-$ RWS $-2=-0$ combined

references

Figure 12. Simulation data of a double lane change maneuver on low friction road surface. 

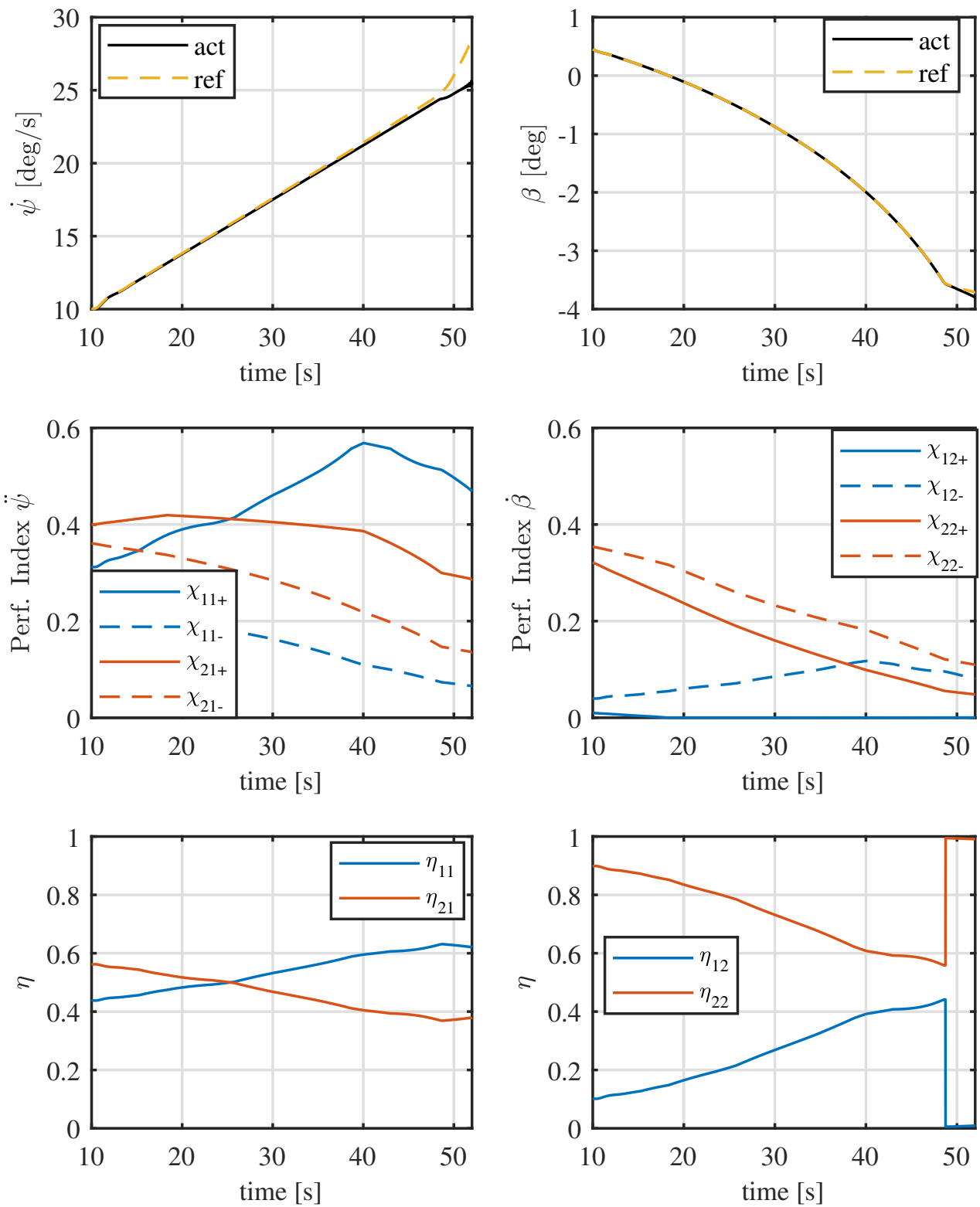

Figure 13. Combined control performance indexes and weighting coefficients in steering pad constant radius maneuver. 

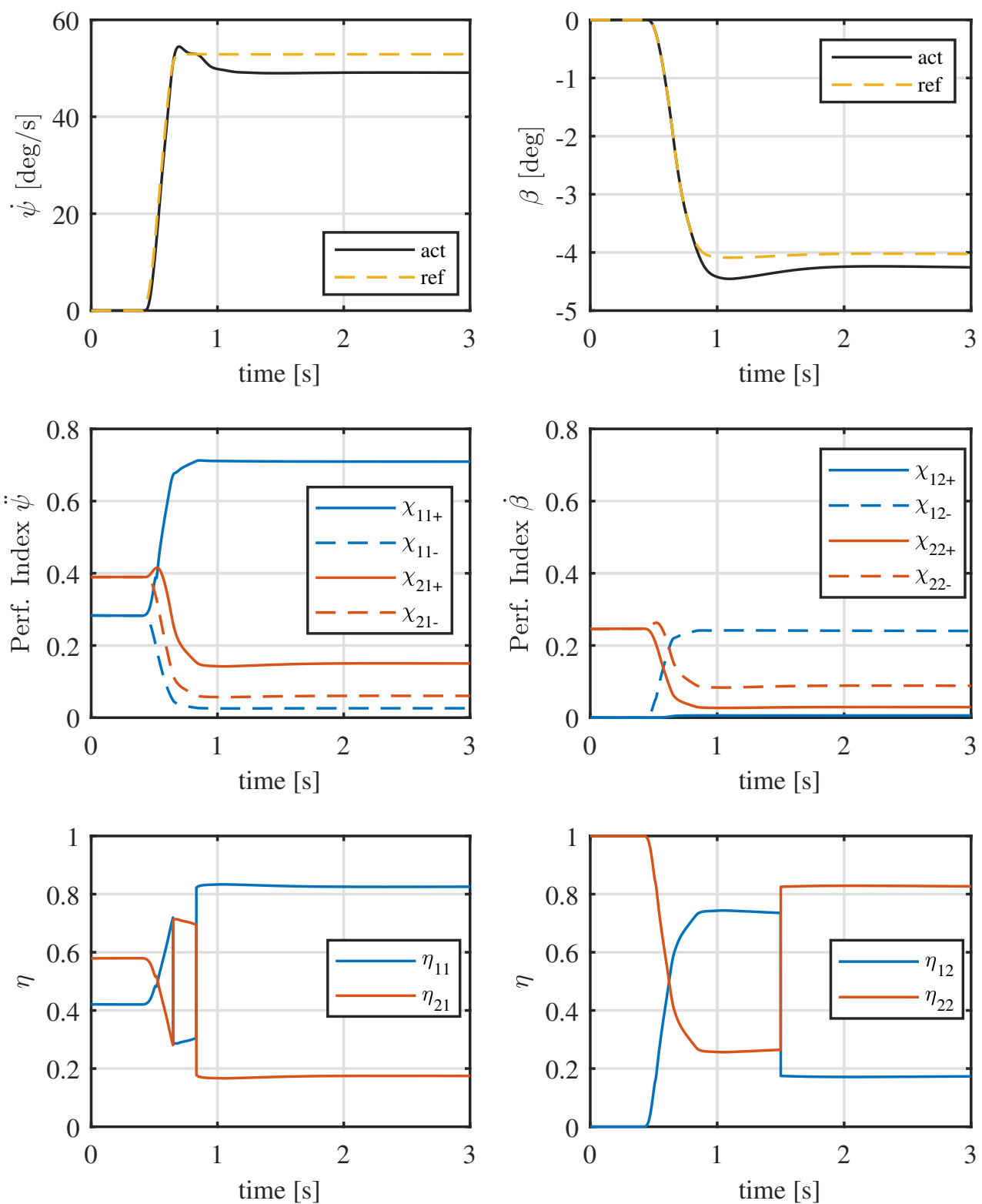

Figure 14. Combined control performance indexes and weighting coefficients in step steer maneuver. 

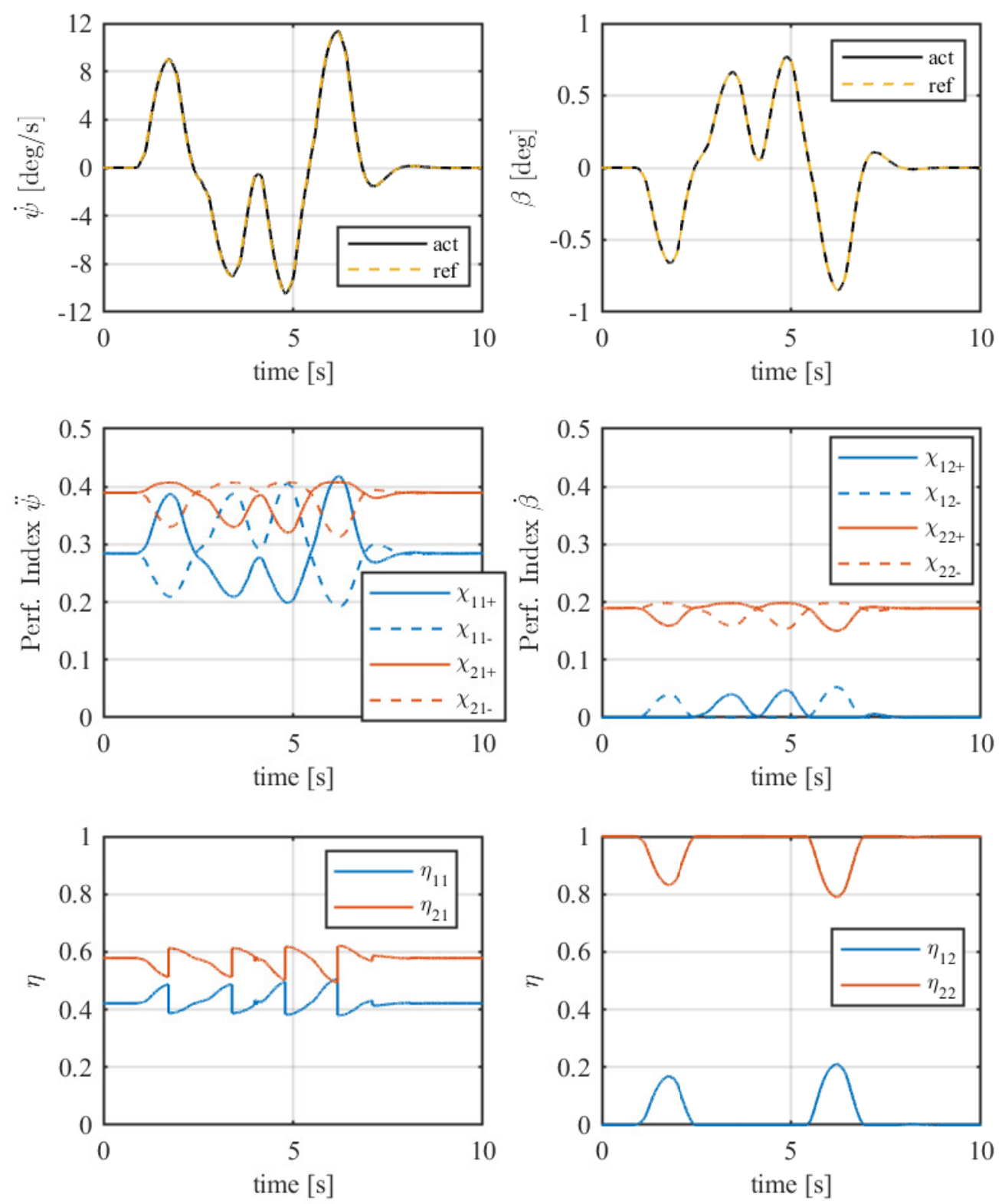

Figure 15. Combined control performance indexes and weighting coefficients in double lane change maneuver. 